\title{
Geochemical Characteristics of the Late Cretaceous Lower Zhoutian Formation in the Jitai Basin, Southeast China: Implications for palaeoenvironment and provenance
}

\section{Kai Yan}

MNR Key Laboratory of Metallogeny and Mineral Assessment, Institute of Mineral Resources, Chinese Academy of Geological Sciences Chun-Lian Wang ( $\square$ wangchunlian312@163.com)

MNR Key Laboratory of Metallogeny and Mineral Assessment, Institute of Mineral Resources, Chinese Academy of Geological Sciences Steffen Mischke

University of Iceland, Faculty of Earth Science

Jiu-Yi Wang

MNR Key Laboratory of Metallogeny and Mineral Assessment, Institute of Mineral Resources, Chinese Academy of Geological Sciences

Li-Jian Shen

MNR Key Laboratory of Metallogeny and Mineral Assessment, Institute of Mineral Resources, Chinese Academy of Geological Sciences Xiao-Can Yu

MNR Key Laboratory of Metallogeny and Mineral Assessment, Institute of Mineral Resources, Chinese Academy of Geological Sciences Ling-Yang Meng

902 Geological brigade of Jianxi Bureau of Exploration and Development for Geology and Mineral Resources

\section{Research Article}

Keywords: paleoclimate, geochemistry

Posted Date: February 18th, 2021

DOI: https://doi.org/10.21203/rs.3.rs-168939/v1

License: @) (1) This work is licensed under a Creative Commons Attribution 4.0 International License. Read Full License

Version of Record: A version of this preprint was published at Scientific Reports on July 5th, 2021. See the published version at https://doi.org/10.1038/s41598-021-93125-8. 


\section{Abstract}

Major, trace and rare earth element geochemistry of the late Cretaceous lower Zhoutian Formation from the Jitai Basin of Southeast China were measured by inductively coupled plasma mass spectromentry (ICP-MS) analysis. The paleoclimate, paleo-environment and provenance of lower Zhoutian Formation in the Jitai Basin are analyzed in detail with these data.According to the research, the range of $\mathrm{Sr} / \mathrm{Cu}$ values is wide, which indicates the changeable palaeoclimate, the curves of $\mathrm{FeO} / \mathrm{MnO}$ and $\mathrm{Al}_{2} \mathrm{O}_{3} / \mathrm{MgO}$ are negatively correlated with the $\mathrm{Sr} / \mathrm{Cu}$ curves. These changes indicate that the palaeoclimate of the late Cretaceous lower Zhoutian Formation in the Jitai Basin was divided into two parts. The lower part experienced two cooling events; the upper part was dominated by warm humid climate.The changes of $\mathrm{B} / \mathrm{Ga}$ and $\mathrm{Sr} / \mathrm{Ba}$ curves are similar to $\mathrm{Sr} / \mathrm{Cu}$ curves. According to these values, the salinity of the late Cretaceous lower Zhoutian Formation in the Jitai Basin was consistent with palaeoclimate. The salinity of lower part changed from salt water to fresh/brackish water. The upper part was mainly fresh/brackish water, and there were many changes from fresh/brackish water to salt water. The values of $\mathrm{Ni} / \mathrm{Co}, \mathrm{V} / \mathrm{Cr}$, $\mathrm{V} /(\mathrm{V}+\mathrm{Ni})$ and $\mathrm{Ce} / \mathrm{Ce}$ are relatively stable, indicating a long-term oxidation environment. The diagrams of La-Th-Sc, Th-Sc-Zr/10, La/Th-Hf and sandstone-siltstone background function show that the provenance in lower Zhoutian Formation mainly is a mixture of upper crust felsic sediments and old components.

\section{Introduction}

As an important carrier of geological information, the geochemical characteristics of clastic rocks record the significant information of provenance, structure, environment and ecological evolution in a real and detailed way. In the process of deposition, the distribution, circulation and differentiation (deficit and enrichment) of trace elements sensitive to redox in water and sediments are not only related to their own chemical properties, but also controlled by the physical and chemical conditions of the deposition medium and palaeoclimate conditions ${ }^{1-4}$. Hence, some major and trace elements that dissolve in water and sensitive to climatic change, which can be used as a valuable proxy of palaeoclimate evolution ${ }^{5}$.

Mesozoic was a period of drastic tectonic changes in South China and even the whole East Asian continent, and it was also a turning point in the development of East Asian tectonics ${ }^{6-10}$. During the late Cretaceous-Paleogene, a series of rift basins were formed in the central part of South China, such as Jianghan Basin, Jitai Basin. At this time, most of the lakes in these Basins evolved into saline lakes and deposited huge amounts of halite and other saline minerals ${ }^{11-}$

12. Deep brine is found in the late Cretaceous strata of Zhoutian Formation in Jitai Basin, which is rich in potassium, lithium, boron, rubidium, cesium, bromine, iodine and other high-value and emerging strategic mineral resources ${ }^{13,14}$.

The formation of deep brine deposits is controlled by material sources, paleoclimatic conditions during the sedimentary period, tectonic conditions of the basin where the water occurs ${ }^{15-17}$. At present, the basic geological research in Jitai Basin is very rare, and the existing data are not enough to study the formation mechanism of deep brine. In order to understand more geological information and characteristics of the late Cretaceous in the Jitai Basin, here we report the geochemical data of major, trace and rare elements of silty mudstone and calcilutite in the late Cretaceous lower Zhoutian Formation. Based on our geochemical data and previous studies, we discuss the palaeoclimate and palaeoenvironment characteristics and provenance of the late Cretaceous lower Zhoutian Formation in the Jitai Basin.

\section{Materials And Methods}

Study area. The Jitai Basin is located in the transition zone between central uplift and southwest depression of Jiangxi province. The basin is about 120 km long and $10-30 \mathrm{~km}$ wide, with an area of about $1850 \mathrm{~km}^{218}$. The basement of the basin varies from region to region. The northern part is dominated by late Paleozoic strata and the southern part by early Paleozoic epimetamorphic series. There is a set of Cretaceous continental red strata in the basin with a thickness of several thousand meters. The lower Cretaceous is exposed at the western edge of the basin. The lower strata are mainly composed of magenta coarse clastic formations, the middle strata are mainly composed of magenta medium fine clastic formations, and the upper strata are mainly composed of argillaceous formations, forming a sedimentary cycle from coarse to fine. The upper Cretaceous is composed of magenta coarse clastic formations and medium fine clastic formations ${ }^{19}$. The lithium-rich ore occurs in the Zhoutian Formation of the late Cretaceous ${ }^{20}$. Since the late Cretaceous, the Jitai Basin began to form in the research area due to the intensification of the Yanshan movement and the strong influence of the fault of Suichuan-Wan'an (Fig. 1).

Sampling and methods. The samples were collected for this research from Well M2 in south Jitai Basin (Fig. 1). A total of 40 core samples from depths between 1100 to $1435 \mathrm{~m}$ were collected from the lower Zhoutian Formation (Table 1). 
Table 1

All samples of the late Cretaceous lower Zhoutian Formation from Well M2, Jitai Basin.

\begin{tabular}{|c|c|c|c|c|c|c|c|c|}
\hline No. & Depth & Lithology & No. & Depth & Lithology & No. & Depth & Lithology \\
\hline M2-1 & $1101 \mathrm{~m}$ & Silty mudstone & M2-15 & $1174 m$ & Silty mudstone & M2-29 & $1315 \mathrm{~m}$ & Silty mudstone \\
\hline M2-2 & $1106 \mathrm{~m}$ & Calcilutite & M2-16 & $1179 m$ & Silty mudstone & M2-30 & $1320 \mathrm{~m}$ & Silty mudstone \\
\hline M2-3 & $1111 \mathrm{~m}$ & Silty mudstone & M2-17 & 1197m & Silty mudstone & M2-31 & $1327 m$ & Calcilutite \\
\hline M2-4 & $1115 \mathrm{~m}$ & Calcilutite & M2-18 & $1205 \mathrm{~m}$ & Calcilutite & M2-32 & $1340 \mathrm{~m}$ & Calcilutite \\
\hline M2-5 & $1121 \mathrm{~m}$ & Calcilutite & M2-19 & $1209 m$ & Calcilutite & M2-33 & $1350 \mathrm{~m}$ & Silty mudstone \\
\hline M2-6 & $1127 m$ & Silty mudstone & M2-20 & $1217 \mathrm{~m}$ & Silty mudstone & M2-34 & $1360 \mathrm{~m}$ & Silty mudstone \\
\hline M2-7 & $1130 \mathrm{~m}$ & Mudstone & M2-21 & $1221 \mathrm{~m}$ & Silty mudstone & M2-35 & $1375 \mathrm{~m}$ & Silty mudstone \\
\hline M2-8 & $1134 m$ & Mudstone & M2-22 & $1245 \mathrm{~m}$ & Silty mudstone & M2-36 & $1386 \mathrm{~m}$ & Calcilutite \\
\hline M2-9 & $1145 \mathrm{~m}$ & Silty mudstone & M2-23 & $1255 \mathrm{~m}$ & Silty mudstone & M2-37 & $1397 m$ & Silty mudstone \\
\hline M2-10 & $1152 m$ & Siltstone & M2-24 & $1270 \mathrm{~m}$ & Silty mudstone & M2-38 & $1405 \mathrm{~m}$ & Calcilutite \\
\hline M2-11 & $1155 \mathrm{~m}$ & Silty mudstone & M2-25 & $1275 \mathrm{~m}$ & Calcilutite & M2-39 & $1415 \mathrm{~m}$ & Calcilutite \\
\hline M2-12 & $1159 m$ & Silty mudstone & M2-26 & $1284 \mathrm{~m}$ & Silty mudstone & M2-40 & $1436 \mathrm{~m}$ & Calcilutite \\
\hline M2-13 & $1164 \mathrm{~m}$ & Silty mudstone & M2-27 & $1295 \mathrm{~m}$ & Silty mudstone & & & \\
\hline M2-14 & 1170m & Silty mudstone & M2-28 & $1306 \mathrm{~m}$ & Silty mudstone & & & \\
\hline
\end{tabular}

X-ray fluorescence spectrometry was used to determine oxides of major elements, such as $\mathrm{SiO}_{2}, \mathrm{TiO}_{2}, \mathrm{Al}_{2} \mathrm{O}_{3}, \mathrm{Fe}_{2} \mathrm{O}_{3}, \mathrm{FeO}, \mathrm{MnO}, \mathrm{MgO}, \mathrm{CaO}, \mathrm{Na} 2 \mathrm{O}, \mathrm{K}_{2} \mathrm{O}, \mathrm{P}_{2} \mathrm{O}_{5}$. $\mathrm{An}$ inductively coupled plasma mass spectrometer (ICP-MS) was used to determine the contents of trace and rare earth element. The samples were fused into glass sheets by alkali fusion and tested on a sequential X-ray fluorescence spectrometer (AB104L, Axios-mAX). The dissolved trace and rare earth elements were measured using an ICP-MS instrument (ELEMENT XR).

All geochemical analysis samples were crushed and ground to less than 200 mesh. The powered samples were first heated and then fused with the mixture of anhydrous lithium tetraborate, lithium fluoride and ammonium nitrate heating in a muffle furnace to determine LOI. The fused samples were heated to $800^{\circ} \mathrm{C}$ for XRF analysis. Calibrations of accuracy and reproducibility were conducted by GB/T 14506.14-2010 and GB/T 14506.28-2010. Powered samples were heated with $\mathrm{HF}$ and $\mathrm{HNO}_{3}$. After evaporated, $\mathrm{HNO}_{3}$ was added to dissolve the samples. And $\mathrm{HNO}_{3}$ was added to the beaker again heating to $130^{\circ} \mathrm{C}$. Finally, the solution was diluted by distilled water for trace analyses, according to GB/T 14506.29-2010 and GB/T 14506.30-2010. And all the experiments were carried out in Analytical Laboratory Beijing Research Institute of Uranium Geology.

\section{Result}

Major elements. The contents of $\mathrm{SiO}_{2}$ are the highest in most sample, but they vary greatly, ranging from $13.48-86.48 \%, \mathrm{Al}_{2} \mathrm{O}_{3}$ contents are between $0.981 \%$ and $31.12 \%, \mathrm{Fe}_{2} \mathrm{O}_{3}$ contents are between $0.685 \%$ and $26.81 \%$ (Table 2). The contents of $\mathrm{CaO}$ and $\mathrm{MnO}$ from silty mudstones in Well $\mathrm{M} 2$ have strong depletion relative to PAAS, but $\mathrm{CaO}$ contents of calcilutites in Well M2 show an obvious enriched. The contents of $\mathrm{Al}_{2} \mathrm{O}_{3}$ and $\mathrm{TiO}_{2}$ from calcilutites are slight depletion. And the $\mathrm{Na}_{2} \mathrm{O}$ contents of all samples are lower than of PAAS and show strong depletion by the plot that normalized to post Archean Australian average shale values (Fig. 2a and b). According to the Table 5, the Sr/Cu values range from 0.99 to 106.56 , with an average of 12.89 . The FeO/MnO values range from 4.15 to 412.22 , with an average of 125.51 . The $\mathrm{Al}_{2} \mathrm{O}_{3} / \mathrm{MgO}$ values range from 0.21 to 63.48 , with an average of 15.73 . The $\mathrm{Mg} / \mathrm{Ca}$ values range from 0.04 to 13.95 , with an average of 3.89 . 
Table 2

The content of major elements from Well M2, Jitai Basin (unit in \%).

\begin{tabular}{|c|c|c|c|c|c|c|c|c|c|c|c|c|c|}
\hline No. & $\mathrm{SiO}_{2}$ & $\mathrm{Al}_{2} \mathrm{O}_{3}$ & $\mathrm{Fe}_{2} \mathrm{O}_{3}$ & $\mathrm{TiO}_{2}$ & Mno & $\mathrm{CaO}$ & $\mathrm{MgO}$ & $\mathrm{K}_{2} \mathrm{O}$ & $\mathrm{Na}_{2} \mathrm{O}$ & $\mathrm{P}_{2} \mathrm{O}_{5}$ & $\mathrm{FeO}$ & LOI & Total \\
\hline M2-1 & 66.74 & 18.34 & 1.56 & 0.786 & 0.008 & 0.577 & 0.834 & 4.36 & 0.327 & 0.036 & 0.65 & 6.34 & 100.6 \\
\hline M2-2 & 20.75 & 8.51 & 4.27 & 0.341 & 0.06 & 30.13 & 2.48 & 2.37 & 0.2 & 0.034 & 1.06 & 30.3 & 100.5 \\
\hline M2-3 & 76.03 & 11.95 & 4.34 & 0.672 & 0.016 & 0.208 & 0.541 & 1.51 & 0.165 & 0.027 & 2.6 & 4.54 & 102.6 \\
\hline M2-4 & 17 & 0.981 & 0.685 & 0.036 & 0.135 & 39.04 & 4.69 & 0.264 & 0.098 & 0.023 & 0.56 & 36.69 & 100.2 \\
\hline M2-5 & 39.39 & 3.7 & 3.56 & 0.101 & 0.138 & 27.28 & 1 & 0.783 & 0.103 & 0.04 & 1.99 & 23.41 & 101.5 \\
\hline M2-6 & 75.32 & 11.22 & 5.13 & 0.68 & 0.014 & 0.153 & 0.72 & 1.8 & 0.179 & 0.022 & 3.6 & 4.35 & 103.2 \\
\hline M2-7 & 43.87 & 28.36 & 9.66 & 1.04 & 0.023 & 0.219 & 1.41 & 4.28 & 0.39 & 0.119 & 6.35 & 10.53 & 106.3 \\
\hline M2-8 & 44 & 14.9 & 26.81 & 0.58 & 0.099 & 0.508 & 2.91 & 0.68 & 0.137 & 0.376 & 19.24 & 8.43 & 118.7 \\
\hline M2-9 & 62.57 & 22.31 & 3.68 & 1.07 & 0.013 & 0.094 & 0.806 & 3.04 & 0.267 & 0.043 & 2.72 & 6.06 & 102.7 \\
\hline M2-10 & 66.59 & 19.79 & 3.76 & 1 & 0.018 & 0.148 & 0.966 & 2.96 & 0.249 & 0.046 & 2.71 & 4.41 & 102.6 \\
\hline M2-11 & 60.71 & 22.1 & 5.45 & 1.04 & 0.02 & 0.138 & 1.36 & 2.79 & 0.229 & 0.036 & 3.87 & 6.07 & 103.8 \\
\hline M2-12 & 69.22 & 17.42 & 3.76 & 0.952 & 0.012 & 0.106 & 0.769 & 2.32 & 0.21 & 0.032 & 2.5 & 5.15 & 102.5 \\
\hline M2-13 & 59.93 & 21.17 & 5.93 & 1.05 & 0.024 & 0.134 & 1.1 & 2.79 & 0.229 & 0.041 & 3.67 & 7.41 & 103.5 \\
\hline M2-14 & 58.36 & 25.79 & 3.94 & 1.15 & 0.013 & 0.107 & 0.742 & 2.99 & 0.232 & 0.051 & 2.72 & 6.57 & 102.7 \\
\hline M2-15 & 50.89 & 28 & 3.95 & 1.1 & 0.008 & 0.116 & 0.699 & 3.57 & 0.292 & 0.044 & 1.37 & 11.3 & 101.3 \\
\hline M2-16 & 64.9 & 21.18 & 3.67 & 0.997 & 0.016 & 0.145 & 0.836 & 2.24 & 0.201 & 0.031 & 2.33 & 5.76 & 102.3 \\
\hline M2-17 & 58.55 & 24.53 & 4.95 & 1.09 & 0.014 & 0.1 & 1.01 & 2.6 & 0.252 & 0.047 & 3.31 & 6.86 & 103.3 \\
\hline M2-18 & 49.1 & 5.62 & 5.75 & 0.61 & 0.318 & 15.38 & 2.38 & 0.967 & 0.111 & 0.028 & 4.88 & 19.26 & 104.4 \\
\hline M2-19 & 24.59 & 8.85 & 13.21 & 0.419 & 0.535 & 17.37 & 5.9 & 1.37 & 0.16 & 0.063 & 11.31 & 27.04 & 110.8 \\
\hline M2-20 & 65.65 & 8.05 & 10.36 & 0.823 & 0.008 & 0.159 & 0.323 & 1.11 & 0.191 & 0.029 & 1.76 & 13.2 & 101.7 \\
\hline M2-21 & 65.17 & 19.01 & 4.77 & 0.934 & 0.025 & 0.12 & 0.914 & 2.18 & 0.211 & 0.035 & 3.46 & 6.56 & 103.4 \\
\hline M2-22 & 49.73 & 29.83 & 4.91 & 1.22 & 0.006 & 0.1 & 0.634 & 3.36 & 0.432 & 0.05 & 2.08 & 9.72 & 102.1 \\
\hline M2-23 & 57.59 & 19.52 & 8.2 & 0.954 & 0.099 & 1.24 & 1.71 & 2.18 & 0.206 & 0.152 & 5.14 & 8.12 & 105.1 \\
\hline M2-24 & 49.86 & 25.77 & 7.19 & 0.998 & 0.037 & 0.632 & 1.53 & 2.95 & 0.33 & 0.13 & 4.75 & 10.53 & 104.7 \\
\hline M2-25 & 13.48 & 4.41 & 6.78 & 0.121 & 0.264 & 38.24 & 2.11 & 0.473 & 0.095 & 0.35 & 5.04 & 33.19 & 104.6 \\
\hline M2-26 & 64.78 & 22.6 & 3.17 & 1.01 & 0.01 & 0.136 & 0.356 & 1.61 & 0.234 & 0.068 & 2.08 & 6 & 102.1 \\
\hline M2-27 & 50.2 & 31.12 & 2.41 & 1.25 & 0.009 & 0.418 & 0.837 & 5.64 & 0.483 & 0.053 & 1.34 & 7.49 & 101.3 \\
\hline M2-28 & 86.46 & 7.08 & 1 & 0.324 & 0.024 & 0.461 & 0.371 & 1.88 & 0.125 & 0.034 & 0.73 & 2.14 & 100.6 \\
\hline M2-29 & 73.71 & 12.58 & 4.33 & 0.568 & 0.069 & 0.933 & 1.34 & 3.39 & 0.147 & 0.084 & 2.46 & 2.37 & 102.0 \\
\hline M2-30 & 76.59 & 12.04 & 2.86 & 0.504 & 0.022 & 0.33 & 1.06 & 3.07 & 0.139 & 0.088 & 2.22 & 2.88 & 101.8 \\
\hline M2-31 & 18.1 & 7.06 & 5.18 & 0.307 & 0.253 & 21.5 & 11.45 & 2.11 & 0.125 & 0.052 & 4.18 & 33.33 & 103.6 \\
\hline M2-32 & 55.15 & 3.53 & 4.78 & 0.249 & 0.257 & 10.92 & 6.16 & 0.645 & 0.079 & 0.047 & 3.89 & 18.13 & 103.8 \\
\hline M2-33 & 80.5 & 7.75 & 2.32 & 0.199 & 0.016 & 1.79 & 1.24 & 1.77 & 0.085 & 0.144 & 1.87 & 3.76 & 101.4 \\
\hline M2-34 & 74.54 & 9.13 & 3.81 & 0.432 & 0.065 & 2.15 & 2 & 2.41 & 0.107 & 0.156 & 2.91 & 5.1 & 102.8 \\
\hline M2-35 & 61.24 & 19.26 & 5.4 & 0.818 & 0.009 & 0.172 & 2.16 & 5.88 & 0.154 & 0.059 & 3.71 & 4.78 & 103.6 \\
\hline M2-36 & 59.29 & 6.75 & 2.82 & 0.342 & 0.211 & 8.17 & 5.17 & 2.06 & 0.098 & 0.184 & 2.47 & 14.86 & 102.4 \\
\hline M2-37 & 72.6 & 12.19 & 3.69 & 0.592 & 0.016 & 1.1 & 1.77 & 3.31 & 0.114 & 0.052 & 2.83 & 4.1 & 102.4 \\
\hline M2-38 & 58.21 & 9.54 & 2.67 & 0.397 & 0.053 & 9.59 & 2.85 & 2.54 & 0.138 & 0.083 & 1.27 & 13.36 & 100.7 \\
\hline M2-39 & 14.57 & 4.7 & 4.77 & 0.131 & 0.141 & 22.26 & 11.52 & 1.65 & 0.078 & 0.056 & 3.63 & 40.03 & 103.5 \\
\hline M2-40 & 70.15 & 11.71 & 2.93 & 0.565 & 0.086 & 1.9 & 1.94 & 3.66 & 0.133 & 0.183 & 2.34 & 6.69 & 102.3 \\
\hline
\end{tabular}


Table 3

The content of trace elements from Well M2, Jitai Basin (unit in ppm).

\begin{tabular}{|c|c|c|c|c|c|c|c|c|c|c|c|c|c|c|c|c|c|c|c|}
\hline No. & Sc & V & $\mathrm{Cr}$ & Co & $\mathrm{Ni}$ & $\mathrm{Cu}$ & $\mathrm{Zn}$ & Ga & $\mathbf{R b}$ & $\mathrm{Sr}$ & $Y$ & Cs & $\mathrm{Ba}$ & $\mathrm{Pb}$ & Th & $\mathbf{U}$ & $\mathrm{Nb}$ & $\mathrm{Ta}$ & $\mathrm{Zr}$ \\
\hline $\begin{array}{l}\text { M2- } \\
1\end{array}$ & 12.9 & 85.8 & 143 & 5.95 & 32.7 & 68.3 & 126 & 24 & 140 & 67.4 & 7.39 & 11.3 & 540 & 34.4 & 15 & 47 & 17.6 & 1.39 & 313 \\
\hline $\begin{array}{l}\text { M2- } \\
2\end{array}$ & 6.84 & 102 & 46.1 & 7.13 & 38.7 & 29.9 & 172 & 11.5 & 75 & 205 & 9.99 & 6.27 & 173 & 26.1 & 7.28 & 3.45 & 7.88 & 0.64 & 92. \\
\hline $\begin{array}{l}\text { M2- } \\
3\end{array}$ & 9.23 & 48.6 & 168 & 9.08 & 23.2 & 13.3 & 42.9 & 14.6 & 48.4 & 45.3 & 13.9 & 3.56 & 450 & 9.4 & 10 & 2.31 & 13.6 & 1.06 & 199 \\
\hline $\begin{array}{l}\text { M2- } \\
4\end{array}$ & 1.27 & 23.4 & 47.5 & 5.28 & 23.2 & 155 & 64.6 & 1.65 & 7.84 & 207 & 9.08 & 0.996 & 18.6 & 24.1 & 1.01 & 0.612 & 0.89 & 0.081 & 14. \\
\hline $\begin{array}{l}\text { M2- } \\
5\end{array}$ & 4.29 & 40.1 & 29.2 & 6.89 & 39.7 & 12.7 & 126 & 5.51 & 19.7 & 117 & 14 & 1.31 & 78.6 & 9.08 & 2.94 & 0.95 & 2.27 & 0.184 & 19 \\
\hline $\begin{array}{l}\text { M2- } \\
6\end{array}$ & 11.7 & 53.5 & 206 & 7.93 & 23.3 & 12.6 & 146 & 14.9 & 56.3 & 34.5 & 14.8 & 3.79 & 290 & 32 & 8.78 & 2.75 & 13.6 & 1.17 & 307 \\
\hline $\begin{array}{l}\text { M2- } \\
7\end{array}$ & 15.9 & 149 & 133 & 29.9 & 53.5 & 24.6 & 113 & 35.5 & 129 & 72.4 & 30.5 & 12.9 & 563 & 34.4 & 19.7 & 4.03 & 22.3 & 1.67 & 233 \\
\hline $\begin{array}{l}\text { M2- } \\
8\end{array}$ & 20.7 & 167 & 122 & 29 & 66 & 16.3 & 236 & 23.6 & 20.6 & 44.1 & 46.9 & 1.46 & 134 & 26.9 & 15.8 & 2.8 & 11 & 0.934 & 155 \\
\hline $\begin{array}{l}\text { M2- } \\
9\end{array}$ & 17 & 86.2 & 188 & 15.1 & 36.3 & 15.7 & 56.5 & 26.2 & 96 & 56.6 & 28.4 & 7.55 & 570 & 9.77 & 17.7 & 3.73 & 21.7 & 1.66 & 343 \\
\hline $\begin{array}{l}\text { M2- } \\
10\end{array}$ & 16.2 & 85 & 154 & 8.6 & 32.3 & 11.1 & 68.9 & 26.3 & 97.6 & 60.1 & 31.6 & 5.44 & 669 & 6.74 & 15.1 & 3.49 & 19.9 & 1.62 & 403 \\
\hline $\begin{array}{l}\text { M2- } \\
11\end{array}$ & 17.5 & 103 & 163 & 14.6 & 39.3 & 15.6 & 90.8 & 31.6 & 95.2 & 49.3 & 18.3 & 8.16 & 638 & 8.56 & 13.2 & 2.83 & 21.6 & 1.69 & 273 \\
\hline $\begin{array}{l}\text { M2- } \\
12\end{array}$ & 14.3 & 78.6 & 194 & 13.3 & 31.5 & 16 & 56.2 & 21.3 & 73.9 & 48.4 & 25.6 & 4.24 & 482 & 10.1 & 13.3 & 3.18 & 18.3 & 1.5 & 290 \\
\hline $\begin{array}{l}\text { M2- } \\
13\end{array}$ & 16.3 & 96.8 & 113 & 22.3 & 38.2 & 28.2 & 71.4 & 27.9 & 88.1 & 57 & 17.4 & 6.2 & 610 & 19.8 & 16 & 3.63 & 20.8 & 1.61 & 381 \\
\hline $\begin{array}{l}\text { M2- } \\
14\end{array}$ & 13.3 & 114 & 145 & 6.87 & 25.7 & 12.8 & 57.5 & 30.5 & 90.7 & 58.1 & 20.2 & 7.91 & 656 & 6.77 & 19 & 3.79 & 24.2 & 1.9 & 317 \\
\hline $\begin{array}{l}\text { M2- } \\
15\end{array}$ & 12.5 & 158 & 138 & 29.5 & 47.4 & 23.7 & 49.8 & 34.7 & 78.1 & 40.7 & 19.2 & 8 & 691 & 39.5 & 12 & 3.75 & 24.4 & 1.84 & 275 \\
\hline $\begin{array}{l}\text { M2- } \\
16\end{array}$ & 10.3 & 109 & 162 & 15 & 35.1 & 10.7 & 64.5 & 26.1 & 70.8 & 38 & 13.3 & 3.93 & 489 & 8.43 & 14.7 & 2.53 & 21.6 & 1.76 & 234 \\
\hline $\begin{array}{l}\text { M2- } \\
17\end{array}$ & 16.8 & 110 & 156 & 16.5 & 62.3 & 20.5 & 88.6 & 34.8 & 94.2 & 64.4 & 34.2 & 5.92 & 707 & 9.04 & 29.8 & 4.36 & 26.4 & 2.06 & 421 \\
\hline $\begin{array}{l}\text { M2- } \\
18\end{array}$ & 7.29 & 49.5 & 140 & 5.9 & 16.9 & 5.42 & 26.2 & 7.48 & 28.8 & 102 & 38.3 & 0.949 & 2562 & 4.75 & 17.9 & 3.72 & 11.4 & 0.856 & 529 \\
\hline $\begin{array}{l}\text { M2- } \\
19\end{array}$ & 12.4 & 62.3 & 63.4 & 12.4 & 28.9 & 8.17 & 36.1 & 11.3 & 41.3 & 73.6 & 26.7 & 2.21 & 308 & 5.3 & 7.14 & 2.61 & 8.98 & 0.665 & 122 \\
\hline $\begin{array}{l}\text { M2- } \\
20\end{array}$ & 8.41 & 55.8 & 217 & 19.5 & 75.3 & 31.2 & 47.6 & 14.2 & 35.7 & 32.2 & 17 & 1.84 & 227 & 99.8 & 15.1 & 3.62 & 15 & 1.39 & 492 \\
\hline $\begin{array}{l}\text { M2- } \\
21\end{array}$ & 14 & 73 & 144 & 11.9 & 34.1 & 14.2 & 145 & 25.3 & 71.3 & 49.9 & 36.7 & 4.55 & 543 & 7.07 & 16.5 & 3.84 & 20.6 & 1.62 & 397 \\
\hline $\begin{array}{l}\text { M2- } \\
22\end{array}$ & 8.77 & 106 & 129 & 26 & 79.4 & 19.1 & 56.9 & 34 & 85.8 & 70.4 & 22.1 & 7.79 & 568 & 32.5 & 18.2 & 5.8 & 28 & 2.06 & 286 \\
\hline $\begin{array}{l}\text { M2- } \\
23\end{array}$ & 134 & 191 & 138 & 28.9 & 61.4 & 19.5 & 98.8 & 27.2 & 42.8 & 64.6 & 16.8 & 4.87 & 517 & 29.3 & 14.2 & 3.35 & 22 & 1.71 & 337 \\
\hline
\end{tabular}




\begin{tabular}{|c|c|c|c|c|c|c|c|c|c|c|c|c|c|c|c|c|c|c|c|}
\hline No. & Sc & V & $\mathrm{Cr}$ & Co & $\mathrm{Ni}$ & $\mathrm{Cu}$ & $\mathrm{Zn}$ & $\mathrm{Ga}$ & $\mathrm{Rb}$ & $\mathrm{Sr}$ & $\mathrm{Y}$ & Cs & $\mathrm{Ba}$ & $\mathrm{Pb}$ & Th & $\mathbf{U}$ & $\mathrm{Nb}$ & $\mathrm{Ta}$ & $\mathrm{Zr}$ \\
\hline $\begin{array}{l}\text { M2- } \\
1\end{array}$ & 12.9 & 85.8 & 143 & 5.95 & 32.7 & 68.3 & 126 & 24 & 140 & 67.4 & 7.39 & 11.3 & 540 & 34.4 & 15 & 47 & 17.6 & 1.39 & 313 \\
\hline $\begin{array}{l}\text { M2- } \\
2\end{array}$ & 6.84 & 102 & 46.1 & 7.13 & 38.7 & 29.9 & 172 & 11.5 & 75 & 205 & 9.99 & 6.27 & 173 & 26.1 & 7.28 & 3.45 & 7.88 & 0.64 & 92.5 \\
\hline $\begin{array}{l}\text { M2- } \\
3\end{array}$ & 9.23 & 48.6 & 168 & 9.08 & 23.2 & 13.3 & 42.9 & 14.6 & 48.4 & 45.3 & 13.9 & 3.56 & 450 & 9.4 & 10 & 2.31 & 13.6 & 1.06 & 199 \\
\hline $\begin{array}{l}\text { M2- } \\
4\end{array}$ & 1.27 & 23.4 & 47.5 & 5.28 & 23.2 & 155 & 64.6 & 1.65 & 7.84 & 207 & 9.08 & 0.996 & 18.6 & 24.1 & 1.01 & 0.612 & 0.89 & 0.081 & 14.7 \\
\hline $\begin{array}{l}\text { M2- } \\
5\end{array}$ & 4.29 & 40.1 & 29.2 & 6.89 & 39.7 & 12.7 & 126 & 5.51 & 19.7 & 117 & 14 & 1.31 & 78.6 & 9.08 & 2.94 & 0.95 & 2.27 & 0.184 & 19 \\
\hline $\begin{array}{l}\text { M2- } \\
6\end{array}$ & 11.7 & 53.5 & 206 & 7.93 & 23.3 & 12.6 & 146 & 14.9 & 56.3 & 34.5 & 14.8 & 3.79 & 290 & 32 & 8.78 & 2.75 & 13.6 & 1.17 & 307 \\
\hline $\begin{array}{l}\text { M2- } \\
7\end{array}$ & 15.9 & 149 & 133 & 29.9 & 53.5 & 24.6 & 113 & 35.5 & 129 & 72.4 & 30.5 & 12.9 & 563 & 34.4 & 19.7 & 4.03 & 22.3 & 1.67 & 233 \\
\hline $\begin{array}{l}\text { M2- } \\
8\end{array}$ & 20.7 & 167 & 122 & 29 & 66 & 16.3 & 236 & 23.6 & 20.6 & 44.1 & 46.9 & 1.46 & 134 & 26.9 & 15.8 & 2.8 & 11 & 0.934 & 155 \\
\hline $\begin{array}{l}\text { M2- } \\
9\end{array}$ & 17 & 86.2 & 188 & 15.1 & 36.3 & 15.7 & 56.5 & 26.2 & 96 & 56.6 & 28.4 & 7.55 & 570 & 9.77 & 17.7 & 3.73 & 21.7 & 1.66 & 343 \\
\hline $\begin{array}{l}\text { M2- } \\
10\end{array}$ & 16.2 & 85 & 154 & 8.6 & 32.3 & 11.1 & 68.9 & 26.3 & 97.6 & 60.1 & 31.6 & 5.44 & 669 & 6.74 & 15.1 & 3.49 & 19.9 & 1.62 & 403 \\
\hline $\begin{array}{l}\text { M2- } \\
11\end{array}$ & 17.5 & 103 & 163 & 14.6 & 39.3 & 15.6 & 90.8 & 31.6 & 95.2 & 49.3 & 18.3 & 8.16 & 638 & 8.56 & 13.2 & 2.83 & 21.6 & 1.69 & 273 \\
\hline $\begin{array}{l}\text { M2- } \\
12\end{array}$ & 14.3 & 78.6 & 194 & 13.3 & 31.5 & 16 & 56.2 & 21.3 & 73.9 & 48.4 & 25.6 & 4.24 & 482 & 10.1 & 13.3 & 3.18 & 18.3 & 1.5 & 290 \\
\hline $\begin{array}{l}\text { M2- } \\
13\end{array}$ & 16.3 & 96.8 & 113 & 22.3 & 38.2 & 28.2 & 71.4 & 27.9 & 88.1 & 57 & 17.4 & 6.2 & 610 & 19.8 & 16 & 3.63 & 20.8 & 1.61 & 381 \\
\hline $\begin{array}{l}\text { M2- } \\
14\end{array}$ & 13.3 & 114 & 145 & 6.87 & 25.7 & 12.8 & 57.5 & 30.5 & 90.7 & 58.1 & 20.2 & 7.91 & 656 & 6.77 & 19 & 3.79 & 24.2 & 1.9 & 317 \\
\hline $\begin{array}{l}\text { M2- } \\
15\end{array}$ & 12.5 & 158 & 138 & 29.5 & 47.4 & 23.7 & 49.8 & 34.7 & 78.1 & 40.7 & 19.2 & 8 & 691 & 39.5 & 12 & 3.75 & 24.4 & 1.84 & 275 \\
\hline $\begin{array}{l}\text { M2- } \\
16\end{array}$ & 10.3 & 109 & 162 & 15 & 35.1 & 10.7 & 64.5 & 26.1 & 70.8 & 38 & 13.3 & 3.93 & 489 & 8.43 & 14.7 & 2.53 & 21.6 & 1.76 & 234 \\
\hline $\begin{array}{l}\text { M2- } \\
17\end{array}$ & 16.8 & 110 & 156 & 16.5 & 62.3 & 20.5 & 88.6 & 34.8 & 94.2 & 64.4 & 34.2 & 5.92 & 707 & 9.04 & 29.8 & 4.36 & 26.4 & 2.06 & 421 \\
\hline $\begin{array}{l}\text { M2- } \\
18\end{array}$ & 7.29 & 49.5 & 140 & 5.9 & 16.9 & 5.42 & 26.2 & 7.48 & 28.8 & 102 & 38.3 & 0.949 & 2562 & 4.75 & 17.9 & 3.72 & 11.4 & 0.856 & 529 \\
\hline $\begin{array}{l}\text { M2- } \\
19\end{array}$ & 12.4 & 62.3 & 63.4 & 12.4 & 28.9 & 8.17 & 36.1 & 11.3 & 41.3 & 73.6 & 26.7 & 2.21 & 308 & 5.3 & 7.14 & 2.61 & 8.98 & 0.665 & 122 \\
\hline $\begin{array}{l}\text { M2- } \\
20\end{array}$ & 8.41 & 55.8 & 217 & 19.5 & 75.3 & 31.2 & 47.6 & 14.2 & 35.7 & 32.2 & 17 & 1.84 & 227 & 99.8 & 15.1 & 3.62 & 15 & 1.39 & 492 \\
\hline $\begin{array}{l}\text { M2- } \\
21\end{array}$ & 14 & 73 & 144 & 11.9 & 34.1 & 14.2 & 145 & 25.3 & 71.3 & 49.9 & 36.7 & 4.55 & 543 & 7.07 & 16.5 & 3.84 & 20.6 & 1.62 & 397 \\
\hline $\begin{array}{l}\text { M2- } \\
22\end{array}$ & 8.77 & 106 & 129 & 26 & 79.4 & 19.1 & 56.9 & 34 & 85.8 & 70.4 & 22.1 & 7.79 & 568 & 32.5 & 18.2 & 5.8 & 28 & 2.06 & 286 \\
\hline $\begin{array}{l}\text { M2- } \\
23\end{array}$ & 134 & 191 & 138 & 28.9 & 61.4 & 19.5 & 98.8 & 27.2 & 42.8 & 64.6 & 16.8 & 4.87 & 517 & 29.3 & 14.2 & 3.35 & 22 & 1.71 & 337 \\
\hline
\end{tabular}


Table 4

The content of rare elements from Well M2, Jitai Basin (unit in ppm).

\begin{tabular}{|c|c|c|c|c|c|c|c|c|c|c|c|c|c|c|c|}
\hline No. & La & $\mathrm{Ce}$ & $\mathrm{Pr}$ & Nd & Sm & Eu & Gd & Tb & Dy & Ho & Er & $\mathrm{Tm}$ & $\mathrm{Yb}$ & Lu & 囚REE \\
\hline M2-1 & 49.9 & 89.8 & 10.3 & 38.2 & 6.77 & 1.31 & 4.5 & 0.538 & 2.3 & 0.295 & 1.33 & 0.184 & 1.42 & 0.247 & 207.09 \\
\hline M2-2 & 20.7 & 38.4 & & 17 & 3.22 & 0.588 & 2.61 & 0.334 & 1.96 & 0.375 & 1.14 & & 1.33 & 0.198 & 92.60 \\
\hline M2-3 & 30.9 & 59.9 & 6.81 & 25.1 & 3.84 & 0.648 & 3.53 & 0.514 & 2.93 & 0.522 & 1.66 & 0.259 & 1.72 & 0.283 & 138.62 \\
\hline M2-4 & 3.2 & 4.88 & 0.787 & 3.4 & 0.85 & 0.242 & 0.894 & 0.209 & 1.21 & 0.281 & 0.76 & 0.139 & 0.836 & 0.113 & 17.80 \\
\hline M2-5 & 7.33 & 13.9 & 1.71 & 7.16 & 1.98 & 0.632 & 2.08 & 0.381 & 2.2 & 0.419 & 1.22 & 0.175 & 1.33 & 0.187 & 40.70 \\
\hline M2-6 & 23.9 & 44.9 & 5.43 & 18.8 & 3.54 & 0.693 & 2.76 & 0.418 & 2.56 & 0.531 & 1.78 & 0.352 & 2.11 & 0.374 & 108.15 \\
\hline M2-7 & 58.6 & 108 & 13.6 & 49.1 & 9.3 & 1.98 & 7.36 & 1.12 & 5.89 & 1.21 & 3.59 & 0.575 & 4.04 & 0.581 & 264.95 \\
\hline M2-8 & 46.3 & 96.6 & 11.5 & 43.6 & 9.4 & 2.15 & 7.73 & 1.42 & 7.85 & 1.64 & 4.59 & 0.747 & 4.6 & 0.635 & 238.76 \\
\hline M2-9 & 53.4 & 96.6 & 11.3 & 39.4 & 6.46 & 1.12 & 5.77 & 0.949 & 5.68 & 1.16 & 3.35 & 0.524 & 3.52 & 0.557 & 229.79 \\
\hline M2-10 & 47.1 & 92.4 & 10.6 & 40.1 & 7.03 & 1.58 & 6.21 & 0.957 & 5.2 & 1.05 & 3.24 & 0.532 & 3.28 & 0.504 & 219.78 \\
\hline M2-11 & 50.4 & 106 & 11.7 & 44.2 & 7.47 & 1.26 & 5.73 & 0.785 & 4.24 & 0.669 & 2.38 & 0.324 & 2.09 & 0.353 & 237.60 \\
\hline M2-12 & 40.8 & 78.2 & 8.4 & 33.9 & 5.86 & 1.26 & 5.16 & 0.875 & 4.76 & 0.933 & 2.44 & 0.399 & 2.75 & 0.423 & 186.16 \\
\hline M2-13 & 49.8 & 92.7 & 11.3 & 39.7 & 6.86 & 1.52 & 5.51 & 0.694 & 3.78 & 0.654 & 2.25 & 0.348 & 2.47 & 0.418 & 218.00 \\
\hline M2-14 & 54.5 & 106 & 13.3 & 47.1 & 8.36 & 1.3 & 5.82 & 0.891 & 4.69 & 0.828 & 2.68 & 0.389 & 2.69 & 0.401 & 248.95 \\
\hline M2-15 & 41.2 & 81.6 & 9.49 & 37.3 & 8.27 & 1.76 & 6.02 & 0.979 & 4.75 & 0.806 & 2.43 & 0.364 & 2.39 & 0.372 & 197.73 \\
\hline M2-16 & 42.6 & 77.4 & 9.45 & 34 & 6.29 & 0.731 & 4.73 & 0.649 & 3.49 & 0.495 & 1.8 & 0.237 & 1.74 & 0.252 & 183.86 \\
\hline M2-17 & 73.9 & 131 & 16.2 & 62.7 & 11.3 & 2.02 & 8.23 & 1.33 & 6.95 & 1.2 & 3.55 & 0.614 & 3.72 & 0.599 & 323.31 \\
\hline M2-18 & 47.1 & 96.7 & 12.7 & 55.7 & 14 & 2.34 & 10.1 & 1.6 & 8.08 & 1.35 & 3.98 & 0.584 & 4.16 & 0.605 & 259.00 \\
\hline M2-19 & 32.6 & 62.2 & 6.95 & 26.8 & 5.22 & 1.69 & 4.82 & 0.867 & 4.7 & 0.974 & 2.86 & 0.442 & 2.93 & 0.419 & 153.47 \\
\hline M2-20 & 41.3 & 77.9 & 8.18 & 28.2 & 4.13 & 0.476 & 4.07 & 0.579 & 3.44 & 0.705 & 2.18 & 0.374 & 2.79 & 0.456 & 174.78 \\
\hline M2-21 & 52.3 & 92 & 11.7 & 41.2 & 7.43 & 1.2 & 6.79 & 1.21 & 6.84 & 1.28 & 3.62 & 0.521 & 3.43 & 0.506 & 230.03 \\
\hline M2-22 & 52.7 & 105 & 12.5 & 44.5 & 8.29 & 2.03 & 6.28 & 0.946 & 4.86 & 0.927 & 2.8 & 0.406 & 2.79 & 0.381 & 244.41 \\
\hline M2-23 & 44 & 88.8 & 10.9 & 41.2 & 7.12 & 1.28 & 5.74 & 0.857 & 4.1 & 0.667 & 2.15 & 0.367 & 2.39 & 0.368 & 209.94 \\
\hline M2-24 & 66 & 123 & 14.4 & 54.3 & 9.82 & 1.82 & 7.49 & 1.06 & 6.03 & 1.06 & 3.2 & 0.5 & 3.61 & 0.503 & 292.79 \\
\hline M2-25 & 17.2 & 33.6 & 3.63 & 15.5 & 2.87 & 1.04 & 2.83 & 0.474 & 2.72 & 0.543 & 1.45 & 0.2 & 1.37 & 0.214 & 83.64 \\
\hline M2-26 & 35.1 & 73.4 & 7.01 & 25.7 & 4.36 & 0.836 & 3.84 & 0.523 & 2.79 & 0.471 & 1.44 & 0.204 & 1.33 & 0.196 & 157.20 \\
\hline M2-27 & 57.4 & 98.2 & 11.8 & 39.3 & 5.96 & 0.773 & 4.84 & 0.553 & 2.66 & 0.407 & 1.59 & 0.246 & 1.93 & 0.334 & 225.99 \\
\hline M2-28 & 18 & 30.9 & 3.3 & 11.8 & 1.95 & 0.343 & 1.67 & 0.24 & 1.15 & 0.216 & 0.741 & 0.13 & 0.942 & 0.134 & 71.52 \\
\hline M2-29 & 43.1 & 78.8 & 9.12 & 33.2 & 5.94 & 1.07 & 4.79 & 0.798 & 4.16 & 0.78 & 2.45 & 0.325 & 2.37 & 0.379 & 187.28 \\
\hline M2-30 & 44.6 & 89.9 & 9.46 & 32.9 & 5.98 & 1 & 4.7 & 0.682 & 3.46 & 0.586 & 1.94 & 0.272 & 1.9 & 0.289 & 197.67 \\
\hline M2-31 & 19.3 & 33 & 4.35 & 16.8 & 3.96 & 1.17 & 3.48 & 0.668 & 3.69 & 0.784 & 2.05 & 0.31 & 2.23 & 0.301 & 92.09 \\
\hline M2-32 & 31 & 55.7 & 6.29 & 23.5 & 5.54 & 0.985 & 4.07 & 0.827 & 4.83 & 0.955 & 2.3 & 0.442 & 2.83 & 0.385 & 139.65 \\
\hline M2-33 & 46.6 & 79.8 & 8.97 & 33 & 6.32 & 1.28 & 5.49 & 0.819 & 4.03 & 0.664 & 1.85 & 0.255 & 1.76 & 0.263 & 191.10 \\
\hline M2-34 & 43.4 & 87.4 & 10.1 & 36.2 & 7.1 & 1.24 & 5.76 & 0.952 & 4.91 & 0.853 & 2.51 & 0.41 & 2.71 & 0.405 & 203.95 \\
\hline M2-35 & 53.2 & 95.1 & 11.1 & 40.5 & 7.16 & 1.42 & 5.23 & 0.9 & 4.68 & 0.853 & 2.73 & 0.422 & 3.14 & 0.481 & 226.92 \\
\hline M2-36 & 34.4 & 65.9 & 7.99 & 29.5 & 6.03 & 1.04 & 5.03 & 0.887 & 4.65 & 0.892 & 2.73 & 0.353 & 2.77 & 0.396 & 162.57 \\
\hline M2-37 & 39.3 & 69.4 & 8.1 & 29.5 & 5.58 & 0.905 & 4.62 & 0.72 & 4.25 & 0.74 & 2.22 & 0.299 & 2.28 & 0.361 & 168.28 \\
\hline M2-38 & 22.1 & 37.9 & 4.68 & 17.1 & 3.25 & 0.691 & 2.94 & 0.507 & 2.75 & 0.651 & 1.7 & 0.264 & 2.08 & 0.29 & 96.90 \\
\hline M2-39 & 17.4 & 33.8 & 4.23 & 16.5 & 4.09 & 0.96 & 3.2 & 0.634 & 3.52 & 0.747 & 1.92 & 0.317 & 1.96 & 0.253 & 89.53 \\
\hline M2-40 & 48 & 92.4 & 12 & 45.2 & 9.05 & 1.71 & 7.53 & 1.27 & 6.4 & 1.24 & 3.32 & 0.534 & 3.64 & 0.538 & 232.83 \\
\hline
\end{tabular}


Table 5

Values of selected elements from Well M2, Jitai Basin.

\begin{tabular}{|c|c|c|c|c|c|c|c|c|c|c|c|c|}
\hline No. & $\mathrm{Sr} / \mathrm{Cu}$ & $\mathrm{FeO} / \mathrm{MnO}$ & $\mathrm{Al}_{2} \mathrm{O}_{3} / \mathrm{MgO}$ & $\mathrm{Mg} / \mathrm{Ca}$ & $\mathrm{B} / \mathrm{Ga}$ & $\mathrm{Sr} / \mathrm{Ba}$ & $\mathrm{Ni} / \mathrm{Co}$ & $\mathrm{V} / \mathrm{Cr}$ & $\mathrm{V} /(\mathrm{V}+\mathrm{Ni})$ & $\mathrm{Ce} / \mathrm{Ce}^{*}$ & $\mathrm{La} / \mathrm{Sc}$ & Th/Sc \\
\hline M2-1 & 0.99 & 81.25 & 21.99 & 1.61 & 3.31 & 0.12 & 5.50 & 0.6 & 0.72 & 0.90 & 3.87 & 1.16 \\
\hline M2-2 & 6.86 & 17.67 & 3.43 & 0.09 & 3.03 & 1.18 & 5.43 & 2.21 & 0.72 & 0.90 & 3.03 & 1.06 \\
\hline M2-3 & 3.41 & 162.5 & 22.09 & 2.89 & 3.56 & 0.10 & 2.56 & 0.29 & 0.68 & 0.94 & 3.35 & 1.08 \\
\hline M2-4 & 1.34 & 4.15 & 0.21 & 0.13 & 1.90 & 11.13 & 4.39 & 0.49 & 0.50 & 0.70 & 2.52 & 0.79 \\
\hline M2-5 & 9.21 & 14.42 & 3.7 & 0.04 & 1.83 & 1.49 & 5.76 & 1.37 & 0.50 & 0.90 & 1.71 & 0.69 \\
\hline M2-6 & 2.74 & 257.14 & 15.58 & 5.23 & 6.03 & 0.12 & 2.94 & 0.26 & 0.70 & 0.90 & 2.04 & 0.75 \\
\hline M2-7 & 2.94 & 276.09 & 20.11 & 7.15 & 1.71 & 0.13 & 1.79 & 1.12 & 0.74 & 0.87 & 3.69 & 1.24 \\
\hline M2-8 & 2.71 & 194.34 & 5.12 & 6.36 & 1.03 & 0.33 & 2.28 & 1.37 & 0.72 & 0.96 & 2.24 & 0.76 \\
\hline M2-9 & 3.61 & 209.23 & 27.68 & 9.53 & 3.00 & 0.10 & 2.40 & 0.46 & 0.70 & 0.90 & 3.14 & 1.04 \\
\hline M2-10 & 5.41 & 150.56 & 20.49 & 7.25 & 3.80 & 0.10 & 3.76 & 0.55 & 0.72 & 0.95 & 2.91 & 0.93 \\
\hline M2-11 & 3.16 & 193.50 & 16.25 & 10.95 & 2.34 & 0.08 & 2.69 & 0.63 & 0.72 & 1.00 & 2.88 & 0.75 \\
\hline M2-12 & 3.03 & 208.33 & 22.65 & 8.06 & 3.54 & 0.10 & 2.37 & 0.41 & 0.71 & 0.96 & 2.85 & 0.93 \\
\hline M2-13 & 2.02 & 152.92 & 19.25 & 9.12 & 3.51 & 0.09 & 1.71 & 0.86 & 0.72 & 0.89 & 3.06 & 0.98 \\
\hline M2-14 & 4.54 & 209.23 & 34.76 & 7.71 & 2.96 & 0.09 & 3.74 & 0.79 & 0.82 & 0.90 & 4.10 & 1.43 \\
\hline M2-15 & 1.72 & 171.25 & 40.06 & 6.70 & 2.31 & 0.06 & 1.61 & 1.14 & 0.76 & 0.94 & 3.30 & 0.96 \\
\hline M2-16 & 3.55 & 145.63 & 25.33 & 6.41 & 3.54 & 0.08 & 2.34 & 0.67 & 0.75 & 0.88 & 4.14 & 1.43 \\
\hline M2-17 & 3.14 & 236.43 & 24.29 & 11.22 & 3.33 & 0.09 & 3.78 & 0.71 & 0.64 & 0.87 & 4.40 & 1.77 \\
\hline M2-18 & 18.82 & 15.35 & 2.36 & 0.17 & 12.5 & 0.04 & 2.86 & 0.35 & 0.75 & 0.90 & 6.46 & 2.46 \\
\hline M2-19 & 9.01 & 21.14 & 1.5 & 0.38 & 2.85 & 0.24 & 2.33 & 0.98 & 0.68 & 0.94 & 2.63 & 0.58 \\
\hline M2-20 & 1.03 & 220 & 24.92 & 2.26 & 12.54 & 0.14 & 3.86 & 0.26 & 0.43 & 0.97 & 4.91 & 1.79 \\
\hline M2-21 & 3.51 & 138.4 & 20.80 & 8.46 & 3.99 & 0.09 & 2.87 & 0.51 & 0.68 & 0.85 & 3.74 & 1.18 \\
\hline M2-22 & 3.69 & 346.67 & 47.05 & 7.04 & 2.63 & 0.12 & 3.05 & 0.82 & 0.43 & 0.94 & 6.01 & 2.08 \\
\hline M2-23 & 3.31 & 51.92 & 11.42 & 1.53 & 3.16 & 0.12 & 2.12 & 1.38 & 0.68 & 0.93 & 0.33 & 0.11 \\
\hline M2-24 & 5.80 & 128.38 & 16.84 & 2.69 & 2.40 & 0.16 & 3.88 & 1.22 & 0.57 & 0.91 & 3.55 & 1.20 \\
\hline M2-25 & 56.22 & 19.09 & 2.09 & 0.06 & 2.64 & 4.81 & 6.97 & 1.74 & 0.76 & 0.97 & 3.47 & 0.62 \\
\hline M2-26 & 8.83 & 208 & 63.48 & 2.91 & 4 & 0.44 & 4.59 & 0.55 & 0.76 & 1.07 & 5.96 & 2.39 \\
\hline M2-27 & 16.86 & 148.89 & 37.18 & 2.22 & 3.20 & 0.42 & 6.03 & 0.86 & 0.52 & 0.86 & 5.57 & 2.18 \\
\hline M2-28 & 4.49 & 30.42 & 19.08 & 0.89 & 14.63 & 0.08 & 2.19 & 0.29 & 0.74 & 0.91 & 7.59 & 2.60 \\
\hline M2-29 & 1.92 & 35.65 & 9.39 & 1.60 & 8.83 & 0.10 & 1.23 & 0.62 & 0.83 & 0.91 & 4.72 & 1.32 \\
\hline M2-30 & 3.24 & 100.91 & 11.36 & 3.57 & 7.45 & 0.18 & 2.83 & 0.68 & 0.88 & 1.00 & 2.92 & 0.58 \\
\hline M2-31 & 39.94 & 16.52 & 0.62 & 0.59 & 6.97 & 2.32 & 3.38 & 1.87 & 0.75 & 0.82 & 2.42 & 0.74 \\
\hline M2-32 & 106.56 & 15.14 & 0.57 & 0.63 & 12.58 & 2.99 & 2.64 & 0.35 & 0.76 & 0.91 & 10.99 & 3.23 \\
\hline M2-33 & 5.94 & 116.88 & 6.25 & 0.77 & 7.70 & 0.36 & 3.33 & 0.36 & 0.83 & 0.89 & 11.51 & 1.93 \\
\hline M2-34 & 3.06 & 44.77 & 4.57 & 1.03 & 12.80 & 0.40 & 5.17 & 0.24 & 0.63 & 0.95 & 7.59 & 2.19 \\
\hline M2-35 & 15.85 & 412.22 & 8.92 & 13.95 & 7.58 & 0.12 & 6.81 & 0.61 & 0.55 & 0.89 & 3.06 & 1.03 \\
\hline M2-36 & 75.68 & 11.71 & 1.31 & 0.70 & 14.00 & 1.93 & 4.96 & 0.33 & 0.74 & 0.91 & 6.53 & 1.86 \\
\hline M2-37 & 5.16 & 176.88 & 6.89 & 1.79 & 7.93 & 0.21 & 2.80 & 0.38 & 0.59 & 0.89 & 3.64 & 1.15 \\
\hline M2-38 & 18.67 & 23.96 & 3.35 & 0.33 & 42.78 & 6.70 & 2.53 & 0.42 & 0.68 & 0.85 & 2.58 & 1.11 \\
\hline M2-39 & 22.30 & 25.74 & 0.41 & 0.58 & 14.51 & 1.81 & 5.07 & 1.06 & 0.55 & 0.90 & 3.29 & 0.89 \\
\hline M2-40 & 25.27 & 27.21 & 6.04 & 1.13 & 13.6 & 0.29 & 2.85 & 0.36 & 0.76 & 0.88 & 5.19 & 1.54 \\
\hline
\end{tabular}


Trace elements. The three elements with the most average content of trace elements are $\mathrm{Ba}, \mathrm{Zr}$ and $\mathrm{Sr}$, and their average contents are $451.20 \mathrm{ppm}, 255.93 \mathrm{ppm}$ and 148.85 ppm respectively (Table 3). According to the plot of trace element compositions normalized against UC (Fig. $2 \mathrm{c}$ and d), the contents of various elements varied greatly. In the plot of silty mudstones normalized to UC, Cr contents are enriched relative to UC in most samples, but Sr contents show strong depletion (Fig. 2c). In Fig. 2d, Cr contents of some calcilutites are enriched. According to the Table 5, the B/Ga values range from 1.03 to 42.78 , with an average of 6.80 . The $\mathrm{Sr} / \mathrm{Ba}$ values range from 0.04 to 11.13 , with an average from 0.99 . The Ni/Co values range from 1.23 to 6.97 , with an average of 3.48 . The $\mathrm{V} / \mathrm{Cr}$ values range from 0.24 to 2.21 , with an average of 0.75 . The $\mathrm{V} /(\mathrm{V}+\mathrm{Ni})$ values range from 0.43 to 0.88 , with an average of 0.70 .

Rare elements. In Table 4, Ce, La and Nd are the three most abundant elements, with Ce content ranging from 4.88 ppm to 131 ppm, La content ranging from $3.2 \mathrm{ppm}$ to $73.9 \mathrm{ppm}$, and Nd content ranging from 3.4 ppm to $62.7 \mathrm{ppm}$. On the chondrite normalized map (Fig. 2e and f), all samples of Zhoutian Formation show a set of steeply dips LREE curves, while the curves of HREE are flat. For the silty mudstone samples, Eu contents are negative anomalies, and curves of Eu are V-shaped in the standardized diagram. For the samples of calcilutite, Eu contents are light negative anomalies. According to the Table 4, the $\otimes R E E$ values range from 17.80 to 323.31 , with an average of 181.14 . According to the Table 5 , the Ce/Ce* values range from 0.70 to 1.07 , with an average of 0.91 . The Th/Sc values range from 0.58 to 3.23 , with an average of 1.31 . The La/Sc values range from 0.33 to 11.51 , with an average of 4.20 .

\section{Discussion}

Palaeoclimate. As one of the methods to distinguish palaeoclimate, element geochemistry, such as $\mathrm{Sr} / \mathrm{Cu}, \mathrm{FeO} / \mathrm{MnO}, \mathrm{Al}_{2} \mathrm{O}_{3} / \mathrm{MgO}$ and $\mathrm{Mg} / \mathrm{Ca}$, has a good indication to distinguish palaeoclimate. The value of $\mathrm{Sr} / \mathrm{Cu}$ is a sensitive indicator of climate, and the high $\mathrm{Sr} / \mathrm{Cu}$ value reflects a hot arid climate, while the low value reflects a warm humid climate. According to the research of Lermanm ${ }^{21}, \mathrm{Sr} / \mathrm{Cu}$ value between 1.3 and 5.0 suggest a warm humid climate, while the value greater than 5.0 indicates a hot arid climate. Mn content is relatively high in the dry environment, but low in the relatively humid conditions, and Fe is easy to be quickly precipitated by $\mathrm{Fe}(\mathrm{OH})$ colloid in the humid environment. So, the high value of $\mathrm{FeO} / \mathrm{MnO}$ in the sediment corresponds to the warm humid climate, and the low value is the response to the hot arid climate. The $\mathrm{Al}_{2} \mathrm{O}_{3} / \mathrm{MgO}$ value in clay minerals and its variation can also reflect the palaeoclimate during deposition, with high values reflecting the warm humid climate and low values indicating the dry climate ${ }^{22}$. The $\mathrm{Mg} / \mathrm{Ca}$ value is also very sensitive to climate change. In general, high values of $\mathrm{Mg} / \mathrm{Ca}$ indicate arid climate, while low values reflect humid climate ${ }^{23}$.

The values of Sr/Cu vary widely, ranging from 0.99 to 106.56 (Table 5 and Fig. 3). This indicates that the Jitai Basin experienced relatively large climatic change during the late Cretaceous period. According to these values and curves, the Cretaceous palaeoclimate in the lower Zhoutian Formation is roughly divided into two parts, $1435-1270 \mathrm{~m}$ and $1270-1100 \mathrm{~m}$ respectively. The values of Sr/Cu in most sections between 1435 and $1270 \mathrm{~m}$ is greater than 5.0 . The values are less than 5.0 at 1321 to $1306 \mathrm{~m}$ and $1360 \mathrm{~m}$. So, the palaeoclimate type in the $1435-1270 \mathrm{~m}$ segment was a hot arid climate, and there was an obvious cooling event between 1321 and $1306 \mathrm{~m}$. The $\mathrm{Sr} / \mathrm{Cu}$ values of the $1100-1270 \mathrm{~m}$ segment are between 1.3 and 5.0 on the whole, and are greater than 5.0 at $1106 \mathrm{~m}, 1121 \mathrm{~m}, 1152 \mathrm{~m}, 1205 \mathrm{~m}$ and $1209 \mathrm{~m}$. Hence, the palaeoclimate type in the $1270-1100 \mathrm{~m}$ segment was mainly warm humid climate, and there was a short period of climate fluctuation in some segments. In Fig. 3, FeO/MnO and $\mathrm{Al}_{2} \mathrm{O}_{3} / \mathrm{MgO}$ curves show similar trend of change, but they are opposite to Sr/Cu curves. Since $\mathrm{FeO} / \mathrm{MnO}$ and $\mathrm{Al}_{2} \mathrm{O}_{3} / \mathrm{MgO}$ are negatively correlated with $\mathrm{Sr} / \mathrm{Cu}$, they all express the same geological meaning. And the change trend of $\mathrm{Mg} / \mathrm{Ca}$ curve is also opposite to that of $\mathrm{Sr} / \mathrm{Cu}$ curve. However, there is usually a positive correlation between $\mathrm{Mg} / \mathrm{Ca}$ and $\mathrm{Sr} / \mathrm{Cu}$, so they are in conflict. Based on the comprehensive analysis of the above data, $\mathrm{FeO} / \mathrm{MnO}, \mathrm{Al}_{2} \mathrm{O}_{3} / \mathrm{MgO}$ and $\mathrm{Sr} / \mathrm{Cu}$ are more accurate indicators of geological significance than $\mathrm{Mg} / \mathrm{Ca}$. According to Table 5 and Fig. 3, the palaeoclimate of the late Cretaceous lower Zhoutian Formation in the Jitai Basin was mainly divided into upper and lower parts. The lower part (1435-1270m) experienced two cooling events and had the palaeoclimate characteristic of wet-dry cycling; the upper part (1270-1100m) was dominated by warm humid climate, which experienced many climatic fluctuations.

Salinity. Boron is one of the most concentrated trace elements in sea water and is mainly attached by illite in coastal to marine environments ${ }^{24,25}$. By comparison, Gallium is principally come from terrestrial environments and is chiefly deposited in the form of smectite. According to studies of Wang et al. ${ }^{26}$ and Lan et al. ${ }^{27}$, the value of $\mathrm{B} / \mathrm{Ga}$ is a reliable indicator of salinity. The value less than 3.0 indicates fresh water, the value ranging between 3.0 and 5.0 indicates brackish water, and the value great than 5.0 indicates salt water ${ }^{28,29}$.

The combination of $\mathrm{SO}_{4}{ }^{2-}$ in the sea/saline water and $\mathrm{Ba}^{2+}$ in the fresh water produces the precipitation of $\mathrm{BaSO}_{4}$. In contrast, the $\mathrm{Sr}$ is assumed to migrate and precipitate in the center of the open marine basin or saline lake as a result of the high solubility of $\mathrm{SrSO}_{4}{ }^{30}$. Therefore, the value of $\mathrm{Sr} / \mathrm{Ba}$ can be used to distinguish marine and terrestrial environments. Overall, the value great than 1.0 usually indicates sea/saline water, and this value less than 1.0 means fresh water $^{31-33}$.

The values of $\mathrm{B} / \mathrm{Ga}$ range from 1.03 to 42.78 by Table 5 . The variation trend of $\mathrm{B} / \mathrm{Ga}$ curves is similar to that of $\mathrm{Sr} / \mathrm{Cu}$ curves, which can be divided into two parts (Fig. 3). One part is 1435-1270m, and all B/Ga values are greater than 5.0 . This indicates a saltwater environment. The other part is $1270-1100 \mathrm{~m}$, and $\mathrm{B} / \mathrm{Ga}$ values are mostly between 3.0 and 5.0, with some less than 3.0 and some great than 5.0 . The salinity of this part is mainly brackish or fresh water, but it has undergone several changes in fresh/brackish and salt water. And the values of Sr/Ba range from 0.04 to 11.13 by Table 5. The values of Sr/Ba are similar to that of $\mathrm{B} / \mathrm{Ga}$ and $\mathrm{Sr} / \mathrm{Cu}$, which are divided into two parts: $1435-1270 \mathrm{~m}$ and $1270-1100 \mathrm{~m}$. Compared with $\mathrm{B} / \mathrm{Ga}$ curve, $\mathrm{Sr} / \mathrm{Ba}$ curve is more closely related to $\mathrm{Sr} / \mathrm{Cu}$ curve (Fig. 3). Through the comparison of $\mathrm{Sr} / \mathrm{Cu}$ and $\mathrm{Sr} / \mathrm{Ba}$ curves, salinity is positively correlated with the change of palaeoclimate in the vertical direction. When the palaeoclimate as a whole was warm humid, the sedimentary water was fresh/brackish, and vice versa. To sum up, the salinity of the late Cretaceous lower Zhoutian Formation in Jitai Basin was mainly divided into upper and lower parts. The salinity of lower part (1435-1270m) changed from salt water to fresh/brackish water with the changes of palaeoclimate. The upper part (1270-1100m) was mainly fresh/brackish water, and there were many changes from fresh/brackish water to salt water. 
Redox condition. Several trace elements, such as $\mathrm{U}, \mathrm{Ni}, \mathrm{V}, \mathrm{Mo}, \mathrm{Cr}, \mathrm{Co}$, are commonly enriched in anoxic sediments because of their solubility is controlled by the redox ${ }^{34-37}$. In the study of late Jurassic redox environment in northwest Europe, Jones et al. ${ }^{38}$ concluded that the values of $\mathrm{Ni} / \mathrm{Co}, \mathrm{V} / \mathrm{Cr}$ and $\mathrm{V} /(\mathrm{V}+\mathrm{Ni}) \mathrm{were}$ very reliable by comparing many parameters, and thus summarized a set of trace indexes for judging the redox environment (Table. 6 ). So, the trace element ratios of $\mathrm{Ni} / \mathrm{Co}, \mathrm{V} / \mathrm{Cr}$ and $\mathrm{V} /(\mathrm{V}+\mathrm{Ni})$ have been broadly used to identify redox conditions $\mathrm{s}^{3,38-41}$.

Likewise, Cerium anomalies in REE (rare earth element) distribution patterns are generally used to study redox conditions, too ${ }^{30,42-45}$. Ce is present as of C $\mathrm{e}^{3+}$ under reducing conditions and separates from other $\mathrm{REE}^{3+}$ in the form of $\mathrm{Ce}^{4+}$ under oxidized condition. The Ce/Ce* value is defined as $2(\mathrm{Ce})_{\mathrm{PAAS}} /\left((\mathrm{La})_{\mathrm{PAAS}}+\right.$ $(\mathrm{Pr})_{\text {PAAS }}$, and PAAS refers to normalization of element concentrations against the Post-Archean Average Australian Shale (PAAS) ${ }^{46}$. The value of Ce/Ce* can sensitively reflect the redox condition in the sedimentary environment. The $\mathrm{Ce} / \mathrm{Ce}{ }^{\star}$ value greater than 1 is a positive anomaly, indicating a reduction environment; while the value less than 0.95 is a negative anomaly, indicating an oxidation environment.

According to the Table 5 , the values of $\mathrm{Ni} / \mathrm{Co}$ range from 1.23 to 6.97 , the values of $\mathrm{V} / \mathrm{Cr}$ range from 0.24 to 2.21 , the values of $\mathrm{V} /(\mathrm{V}+\mathrm{Ni})$ range from 0.43 to 0.88 , the value of $\mathrm{Ce} / \mathrm{Ce}$ range from 0.70 to 1.07 . All $\mathrm{Ni} / \mathrm{Co}$ values are less than 7.0 , indicating an oxidation/weak oxidation environment. The $\mathrm{Ni} / \mathrm{Co}$ value of lower part (1435-1270m) varies greatly, with the maximum value of 6.97 , but it is still in a weak oxidation environment. The Ni/Co value of upper part (1270$1100 \mathrm{~m}$ ) is relatively stable, which is greater than 5.0 at $1121 \mathrm{~m}, 1106 \mathrm{~m}$ and $1101 \mathrm{~m}$ only. All $\mathrm{V} / \mathrm{Cr}$ values were less than 2.0 except at $1106 \mathrm{~m}$, which were in an oxidation environment. Most of the $\mathrm{V} /(\mathrm{V}+\mathrm{Ni})$ values are between 0.6 and 0.77 , and only four are great than 0.77 , of which three are in the lower part $(1350 \mathrm{~m}$, $1320 \mathrm{~m}$ and $1315 \mathrm{~m}$ ). Most of the $\mathrm{Ce} / \mathrm{Ce}^{\star}$ values are around $0.95,1.07$ at $1284 \mathrm{~m}$ and 0.70 at $1115 \mathrm{~m}$. Compared with Table 5, Table 6 and Fig. 3, all values indicate that the late Cretaceous lower Zhoutian Formation was in oxidized environment, in which there were some small fluctuation in lower part (1435$1270 \mathrm{~m})$.

Table 6

Geochemical indicates of redox environment.

\begin{tabular}{|lllll|}
\hline Palec-oxygenation Facies & Oxygen Content $(\mathrm{mL} / \mathrm{L})$ & $\mathrm{V} / \mathrm{Cr}$ & $\mathrm{Ni} / \mathrm{Co}$ & $\mathrm{V} /(\mathrm{V}+\mathrm{Ni})$ \\
\hline Anaerobic, extremely dysaerobic & $<0.2$ & $>4.25$ & $>7.0$ & $>0.77$ \\
Dysaerobic, secondary aerobic & $0.2-2.0$ & $2.0-4.25$ & $5.0-7.0$ & $0.60-0.77$ \\
\hline Aerobic & $>2.0$ & $<2.0$ & $<5.0$ & $<0.6$ \\
\hline
\end{tabular}

Provenance. The chemical composition of terrigenous clastic rocks is a comprehensive reflection of the nature, denudation and transport of the source area. During these processes, elements of $\mathrm{Cs}, \mathrm{Zr}$, Th, Hf, Ti, La and $\mathrm{Yb}$ have good stability, even during weathering, transportation and diagenesis. Because of their non-migration, they are often used to evaluate the tectonic setting and the type of source rock in source area ${ }^{46-50}$. Rare earth elements change slightly during deposition. The REE abundance in source rocks and the weathering conditions in source area are the major factors controlling the REE in sediments. Hence, $\mathrm{REE}$ in clastic sedimentary rocks are widely used as the main indicator to identify the provenance ${ }^{46,51}$. In general, $\mathrm{La}-\mathrm{Th}-\mathrm{Sc}, \mathrm{Th}-\mathrm{Sc}-\mathrm{Zr} / 10$ and $\mathrm{La} / \mathrm{Th}-\mathrm{Hf}$ can be used to identify the tectonic environment of the source area ${ }^{48,52-54}$.

The La-Th-Sc discriminant diagram can better distinguish the continental island arc, while the Th-Sc-Zr/10 discriminant diagram can distinguish the active continental margin from the passive continental margin. In Th-Sc-Zr/10 discriminant diagram, the samples are mainly located in the continental island arc, some in the passive margin, and a few points not in any region (Fig. 4a). In La-Th-Sc discriminant diagram, most of the samples are located in the continental island arc, some in the region of active/passive continental margin, and only one sample in the oceanic island arc (Fig. 4b). In La/Th-Hf discriminant diagram, most of the samples are located in the areas of increasing old sediment component, some in the regions of felsic source and felsic/basic source, and only one sample in the andesitic arc source (Fig. 5). And the values of Th/Sc range from 0.58 to 3.23, with an average of 1.31 (Table 5 ). Most of Th/Sc values are located in the range of felsic rocks (0.84-20.5) and are much larger than mafic rocks $(0.05-0.22)$. The values of La/Sc range from 0.33 to 11.51 , with an average of 4.20 (Table 5). Like the La/Sc values, Th/Sc values mostly lie within the felsic rock range (2.5-16.3) ${ }^{55-57}$. Hence, most of the samples are plotted in the continental island arc, some in the passive margin. The sediments mainly come from the provenance of the mixing of felsic material in the continental upper crust with old sediments.

\section{Conclusions}

This study represents a first relatively systematic study on major, trace and rare elements of the late Cretaceous lower Zhoutian Formation in the Jitai Basin. The main conclusions are as follows:

The changes of palaeoclimate were mainly divided into upper and lower parts. The lower part (1435-1270m) was dominated by hot arid climate, and a cooling event occurred. And the upper part (1270-1100m) was dominated by warm humid climate, which experienced many climatic fluctuations.

The salinity of lower part (1435-1270m) changed from salt water to fresh/brackish water with the changes of palaeoclimate. The upper part (1270-1100m) was mainly fresh/brackish water, and there were many changes from fresh/brackish water to salt water. And the late Cretaceous lower Zhoutian Formation was in oxidized environment, in which the lower part (1435-1270m) had some small fluctuation.

The lower Zhoutian Formation in the Jitai Basin shows a major tectonic setting of continental island arc, follows by continental margin. And its provenance is a mixture of upper crust felsic sediments and old sediment components. 


\section{Declarations}

\section{Acknowledgements}

This study was supported by Welfare Basic Scientific Research Business Expenses (Nos. KK2005 and KK2016), the National Basic Research Program of China (973 Program) (No. 2011CB403007), the China Geological Survey (No. DD20190606). We acknowledge 902 Geological brigade of Jianxi Bureau of Exploration and Development for Geology and Mineral Resources for obtaining data of geology.

\section{Author contributions}

K.Y. and C.L.W. designed the research in the manuscript. S.M., J.Y.W. and L.J.S. revised the manuscript. X.C.Y. and L.Y.M. participated in sample collection and processing.

\section{Competing interests}

The authors declare no competing interests.

\section{Additional information}

Correspondence and requests for materials should be addressed to C.L.W.

\section{References}

1. Nameroff, T.J., Calvert, S.E. \& Murray, J.W. Glacial-interglacial variability in the eastern tropical North Pacific oxygen minimum zone recorded by redoxsensitive trace metals. Paleoceanography 19, 1010 (2004).

2. Tribovillard, N., Averbuch, O., Deleeschouwer, X., Racki, G. \& Riboulleau, A. Deep-water anoxia over the Frasnian-Famennian boundary (La Serre, France): a tectonically-induced oceanic anoxic event? Terra Nova 16, 288-295 (2004).

3. Tribovillard, N., Algeo, T.J., Lyons, T. \& Riboulleau, A. Trace metals as paleoredox and paleoproductivity proxies: An update. Geol. 232, 12-32 (2006).

4. Zhang, L. et al. Geochemistry of sediments from the Huaibei Plain (east China): Implications for provenance, weathering, and invasion of the Yellow River into the Huaihe River. Asian Earth Sci. 121, 72-83 (2016).

5. Wei, G.J., Liu, Y., Li, X.H., Shao, L.\& Fang, D.Y. Major and trace element variations of the sediments at ODP Site 1144, South China Sea, during the last 230 ka and their palaeoclimate implications. Palaeoclimatol. Palaeoecol. 212, 331-342 (2004).

6. Ren, J.S. On the geotectonics of southern China. Acta Geol. Sin-Engl. 4, 111-136 (1991).

7. Zhao, Y. et al. Yanshanian movement and conversion oftectonic regimes in East Asia. Earth Sci. Front. 11, 319-328 (2004).

8. Dong, S.W. et al. Jurassic tectonic revolution in China and new interpretation of the "Yanshan movement". Acta Geol. Sin. 82, 334-347 (2008).

9. Dong, S.W. et al. The tectonic stress field in the Dabashan orogen resulting from late Mesozoic intra-continental orogeny. Acta Geol. Sin. 31, 769-780 (2010).

10. Rodríguez-López, J.P. \& Wu, C.H. Recurrent deformations of aeolian desert dunes in the Cretaceous of the South China Block: trigger mechanisms variability and implications for aeolian reservoirs. Pet. Geol. 119, 104483 (2010).

11. Fang, Z.X. Sedimentary filling model of salt lake in the Janghan Basin (Petroleum Industry Press, 2006).

12. Yao, Q.C.\& Lou, J.S. An analysis of hydrocarbon pooling conditions in Yuanjiang sag, the Dongting basin. Gas Ind. (Chengdu, China) 28, 37-40 (2008).

13. Liu, C.L., Wang, M.L., Jiao, P.C.\& Chen, Y.Z. The probing of regularity and controlling factors of potash deposits distribution in Lop Nur salt lake, Xinjiang Acta Geosci. Sin. 30, 796-802 (2009)

14. Liu, C.L. Characteristics and formation of potash deposits in continental rift basins: a review. Acta Geosci. Sin. 5, 515-527 (2013).

15. Liu, C.L. et al. Studies of fluid inclusions in glauberite of middle upper Pleistocene strata and their paleoclimatic significance in lop Nur salty lake, Xinjiang, NW China. Acta Mineral. Sin. 1, 93-98 (2006).

16. Liu, C.L., Jiao, P.C. \& Wang, M.L. A tentative discussion on exploration model for potash deposits in basins of China. Deposits (Beijing, China) 4, 581-592 (2010).

17. Sun, X.H. et al. Paleoclimatic information recorded in fluid inclusions in halites from Lop Nur, Western China. Rep. 7, 1-9 (2017).

18. Yu, X.Q., Shu, L.S., Deng, G.H., Wang, B. \& Zu, F.P. Geochemical Features and tectonic significance of the alkaline-basalts from Ji'an-Taihe Basin, Jiangxi province. Geoscience (Beijing, China) 1, 133-140 (2005).

19. Lu, Q.Y. Sedimentary characteristics of Cretaceous in the Taihe depression, Jitai Basin, Jiangxi. Geochem. Explor. 5, 395-398 (1991).

20. Zhou, M.J., Hu, L., Huang, X.N. \& Han, X.P. Metallogenic geological characteristics and prospect of development and utilization of Meigang Li-bearing Brine deposit in the Taihe County, Jiangxi Province. Min. 33, 61-64 (2017).

21. Lerman, A. Lakes: Chemistry, Geology, Physics (Springer, New York, 1978).

22. Liu, G. \& Zhou, D.S. Application of micro elements analysis in identifying sedimentary environment. Pet. Geol. 29, 307-314 (2007).

23. Song, M.S. Sedimentary environment geochemistry in the Shasi section of southern ramp, Donying depression. Mineral. Petrol. 25, 67-73 (2005).

24. Couch, E.L. Calculation of paleosalinities from boron and clay mineral data. Assoc. Pet. Geol. Bull. 55, 1829-1837 (1971). 
25. Dominik, J. \& Stanley, D.J. Boron, beryllium and sulfur in Holocene sediments and peats of the Nile delta, Egypt: their use as indicators of salinity and climate. Geol. 104, 203-216 (1993).

26. Wang, Y.Y., Guo, W.Y. \& Zhang, G.D. Application of some pollen spore assemblage and its significance of stratigraphy and paleogeographic change in the Yangtze delta. Oceanologia 6, 28-38 (1979).

27. Lan, H.X., Ma, D.X., Xu, M.G., Zhou, Q.W. \& Zhang, G.W. Some geochemical indicators of the Pearl River Delta and their facies significance. Geol. Quat. Geol. 7, 39-49 (1987).

28. Hu, X.F. et al. Trace element characteristics of Eocene Jijuntun Formation andthe favorable metallogenic conditions of oil shale in Fushun Basin. Jilin Univ., Earth Sci. Ed. 42, 60-71 (2012).

29. Zhang, M.M., Liu, Z.J., Xu, S.C., Sun, P.C. \& Hu, X.F. Element response to the ancient lake information and its evolution history of argillaceous source rocks in the Lucaogou Formation in Sangonghe Area of Southern Margin of Junggar Basin. Earth Sci. 24, 987-996 (2013).

30. Cao, J. et al. Trace and rare earth element geochemistry of Jurassic mudstones in the northern Qaidam Basin, northwest China. Erde 72, 245-252 (2012).

31. Deng, H.W. \& Qian, K. Sedimentary geochemistry and environmental analysis (Lanzhou: Gansu Science and Technology Press ,1993).

32. Wang, A.H. Discriminant effect of sedimentary environment by the Sr/Ba ratio of different existing forms. Acta Sedimentol. Sin. 4, 169-174 (1996).

33. Peng, S.Z. et al. Geochemical and grain-size evidence for the provenance of loess deposits in the Central Shandong Mountains region, northern China. Res. 85, 290-298 (2016).

34. Francois, R. The study on the regulation of the concentrations of some trace metals (Rb, Sr, Zn, Cu, V, Cr, Ni, Mn and Mo) in Saanich inlet sediments, British Columbia, Canada. Geol. 83, 285-308 (1988).

35. Arthur, M.A. \& Sageman, B.B. Marine black shales: depositional mechanisms and environments of ancient deposits. Rev. Earth Planet. Sci. 22, 499-551 (1994).

36. Yuri, Z.N., Eder, V.G. \& Zamirailova, A.G. Composition and formation environments of the Upper Jurassic-Lower Cretaceous black shale Bazhenov Formation (the central part of the West Siberian Basin). Pet. Geol. 25, 289-306 (2008).

37. Hetzel, A., März, C., Vogt, C. \& Brumsack, H.J. Geochemical environment of Cenomanian-Turonian black shale deposition at Wunstorf (northern Germany). Cretaceous Res. 32, 480-494 (2011).

38. Jones, B. \& Manning, D.A.C. Comparion of geochemical indices used for the interpretation of palaeoredox conditions in ancient mudstones. Geol. 111, 111-129 (1994).

39. Dill, H. Metallogenesis of early Paleozoic graptolite shales from the Graefenthal Horst (Northern Bavaria-Federal Republic of Germany). Geol. 81, 889903 (1986).

40. Calvert, S.E. \& Pedersen, T.F. Geochemistry of recent oxic and anoxic marine sediments: implications for the geological record. Geol. 113, 67-88 (1993).

41. Algeo, T.J. \& Maynard, J.B. Trace-element behavior and redox facies in core shales of upper Pennsylvanian Kansas-type cyclothems. Geol. 206, 289318 (2004).

42. Zhao, Z.H. Principles of Trace Element Geochemistry (Science Press, Beijing, 1997).

43. Holser, W.T. Evaluation of the application of rare-element elements to paleoceanography. Palaeoclimatol. Palaeoecol. 132, 309-323 (1997).

44. Shields, G. \& Stille, P. Diagenetic constraints on the use of cerium anomalies as palaeoseawater redox proxies: an isotopic and REE study of Cambrian phosphorites. Geol. 175, 29-48 (2001).

45. Teng, G., Liu, W.H., Xu, Y.C. \& Chen, J.F. Correlative study on parameters of inorganic geochemistry and hydrocarbon source rocks formative environment. Earth Sci. 20, 193-200 (2005).

46. Taylor, S.R. \& McLennan, S.M. The continental crust: Its composition and evolution, an examination of the geochemical record preserved in sedimentary rocks (Oxford: Blackwell, Oxford University, 1985).

47. McLennan, S.M. Rare earth elements in sedimentary rocks: influence of provenance and sedimentary processes. Mineral. Geochem. 21, 169-200 (1989).

48. Bhatia, M.R. \& Crook, K.A. Trace element characteristics of graywackes and tectonic setting discrimination of sedimentary basins. Mineral. Petrol. $\mathbf{9 2}$, 181-193 (1986).

49. Girty, G.H., Hanson, A.D., Knaack, C. \& Johnson, D. Provenance determined by REE, Th, and Sc analyses of metasedimentary rocks, Boyden Cave roof pendant, central Sierra Nevada, California. Sediment. Res. 64, 68-73 (1994).

50. Garver, J.I. \& Scott, T.J. Trace elements in shale as indicators of crustal provenance and terrane accretion in the southern Canadian Cordillera. Soc. Am. Bull. 107, 440-453 (1995).

51. Bhatia, M.R. \& Taylor, S.R. Trace-element geochemistry and sedimentary provinces: a study from the Tasman Geosyncline, Australia. Geol. $\mathbf{3 3}, \mathbf{1 1 5 - 1 2 5}$ (1981).

52. Bhatia, M.R. Plate tectonics and geochemical composition of sandstones. Geol. 91, 611-627 (1983).

53. Floyd, P. A., Leveridge, B.E. Tectonic environment of the Devonian Gramscatho basin, south Cornwall: Framework mode and geochemical evidence from turbidite sandstones. Geol. Soc. (London, U. K.) 144, 531-542 (1987).

54. Shao, L., Stattegger, K. \& Garbe-Schoenberg, C. Sandstone Petrology and Geochemistry of the Turpan Basin (NW China): Implications for the Tectonic Evolution of a Continental Basin. Sediment. Res. 71,37-49 (2001).

55. Cullers, R.L. The controls on the major and trace element variation of shales, siltstones, and sandstones of Pennsylvanian-Permian age from uplifted continental blocks in Colorado to platform in Kansas, USA. Cosmochim. Acta 58, 4955-4972 (1994). 
56. Cullers, R.L. The geochemistry of shales, siltstones and sandstones of Pennsylvanian-Permian age, Colorado, USA: implications for provenance and metamorphic studies. Lithos 51, 181-203 (2000).

57. Cullers, R.L., Podkovyrov, V.N. Geochemistry of the Mesoproterozoic Lakhanda shales in southeastern Yakutia, Russia: implications for mineralogical and provenance control, and recycling. Precambrian Res. 104, 77-93 (2000).

\section{Figures}

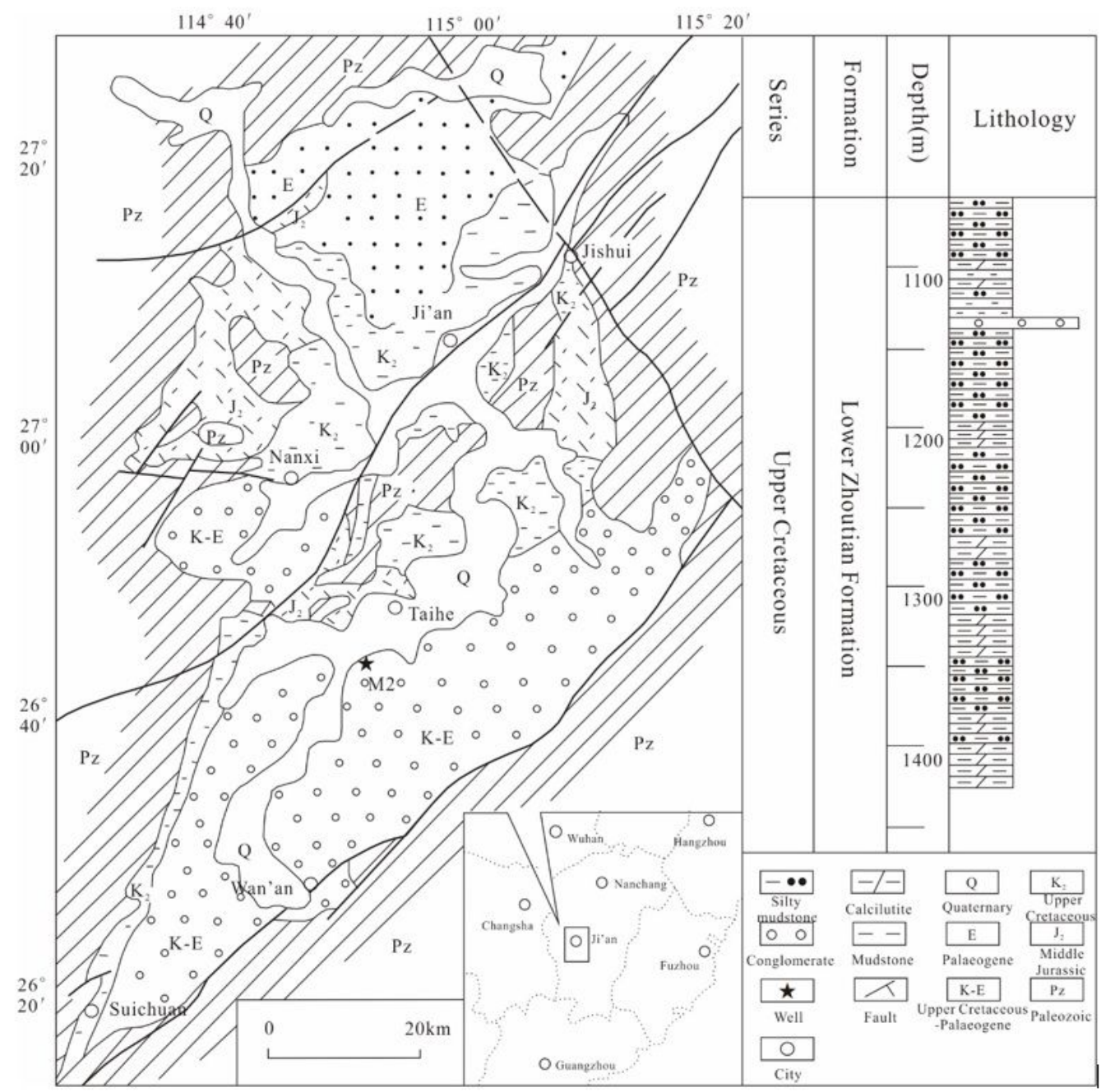

\section{Figure 1}

Regional tectonic map of Jitai Basin, Southeast China. Note: The designations employed and the presentation of the material on this map do not imply the expression of any opinion whatsoever on the part of Research Square concerning the legal status of any country, territory, city or area or of its authorities, or concerning the delimitation of its frontiers or boundaries. This map has been provided by the authors. 

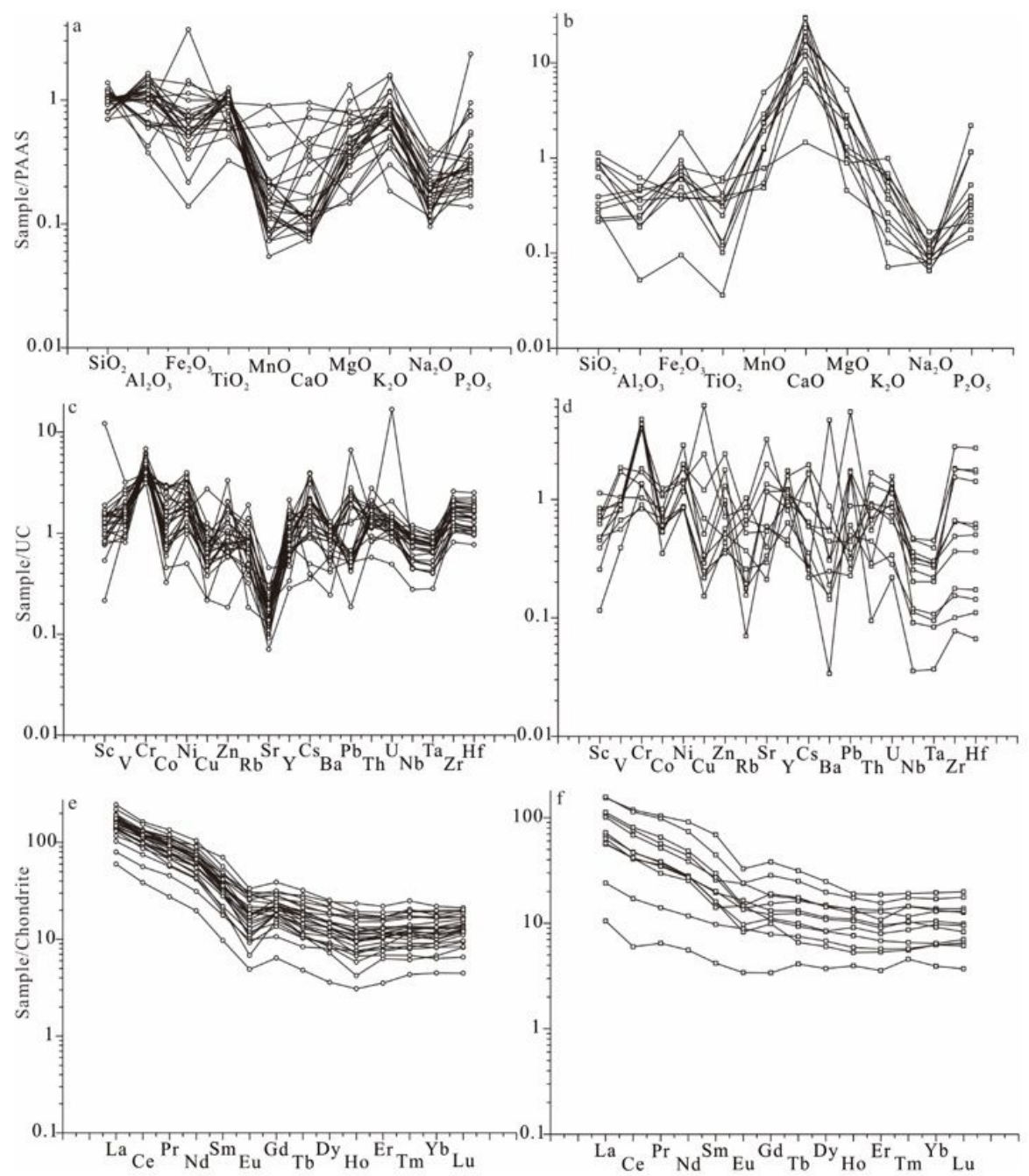

Figure 2

(a) PAAS-normalized major-element diagram of silty mudstones in Well M2. (b) PAAS-normalized major-element diagram of calcilutites in Well M2. (c) UCnormalized trace-element diagram of silty mudstones in Well M2. (d) UC-normalized trace-element diagram of calcilutites in Well M2. (e) Chondrite-normalized rare-element diagram of silty mudstones in Well M2. (f) Chondrite-normalized rare-element diagram of calcilutites in Well M2. Data from Table 2, Table 3 and Table 4. 


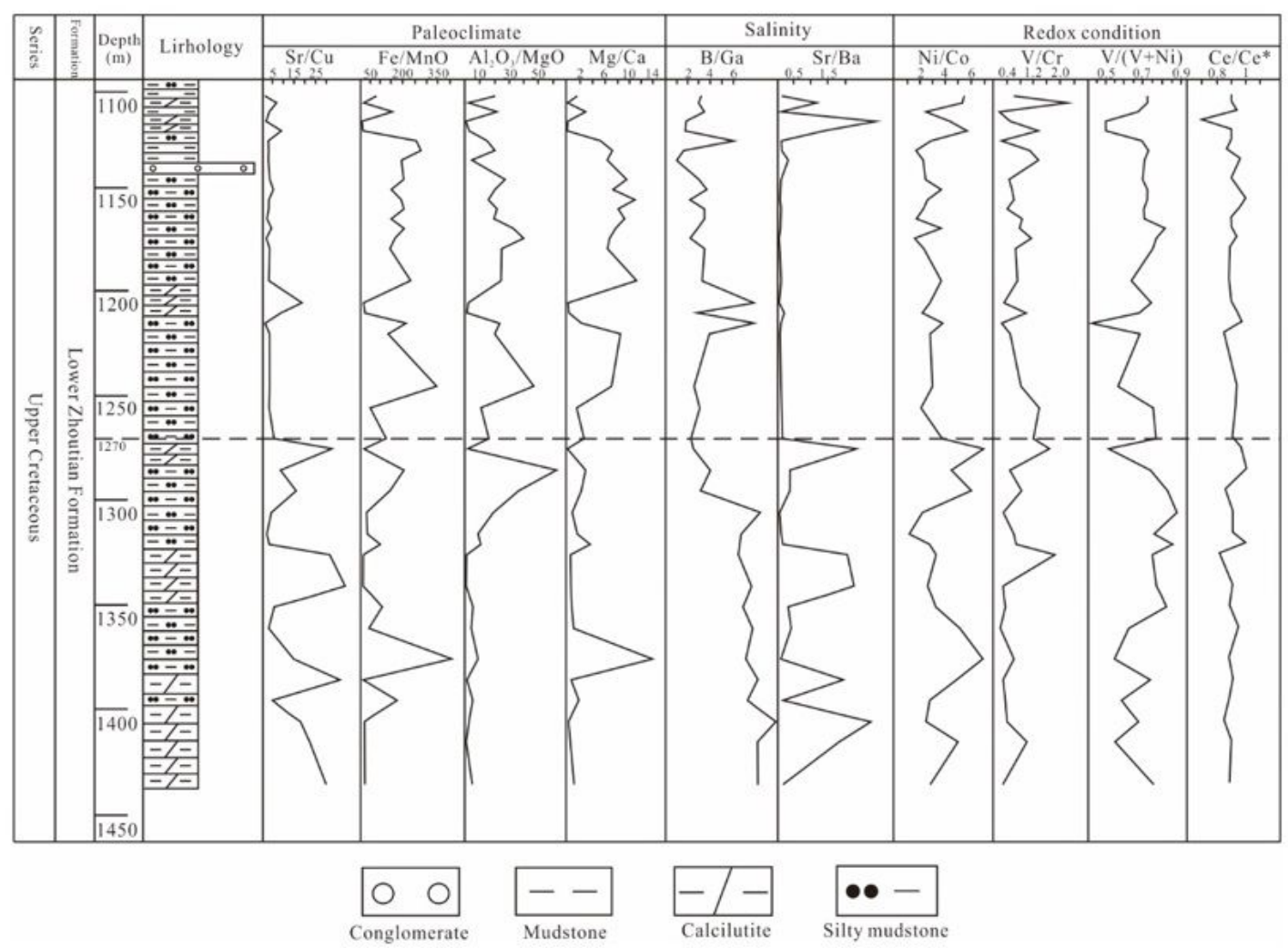

Figure 3

Geochemical indicates of elements value from lower Zhoutian Formation in the Jitai Basin, Southeast China.

a

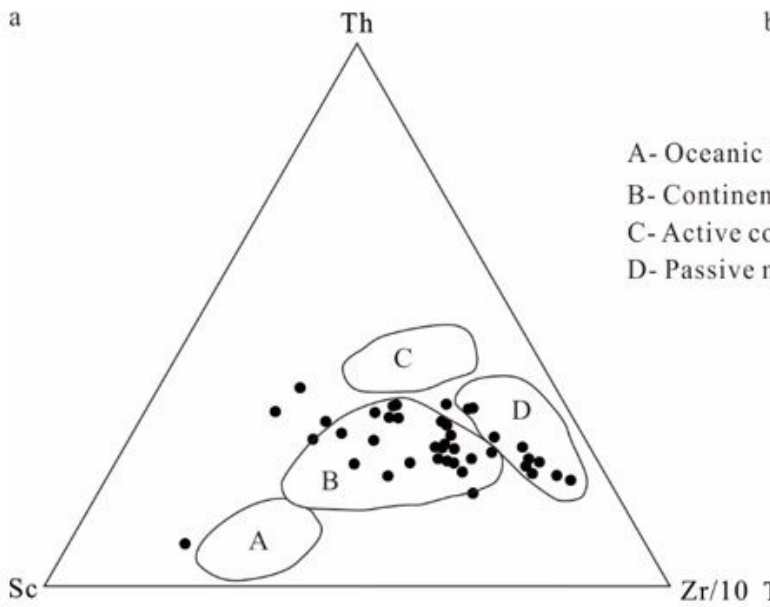

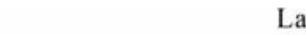

Figure 4

(a) Th-Sc-Zr/10 discriminatory plot (modified after Bhatia \& Crook48) of late Cretaceous samples from the lower Zhoutian Formation, Jitai Basin. (b) La-Th-Sc discriminatory plot (modified after Bhatia \& Crook48) of late Cretaceous samples from the lower Zhoutian Formation, Jitai Basin. 


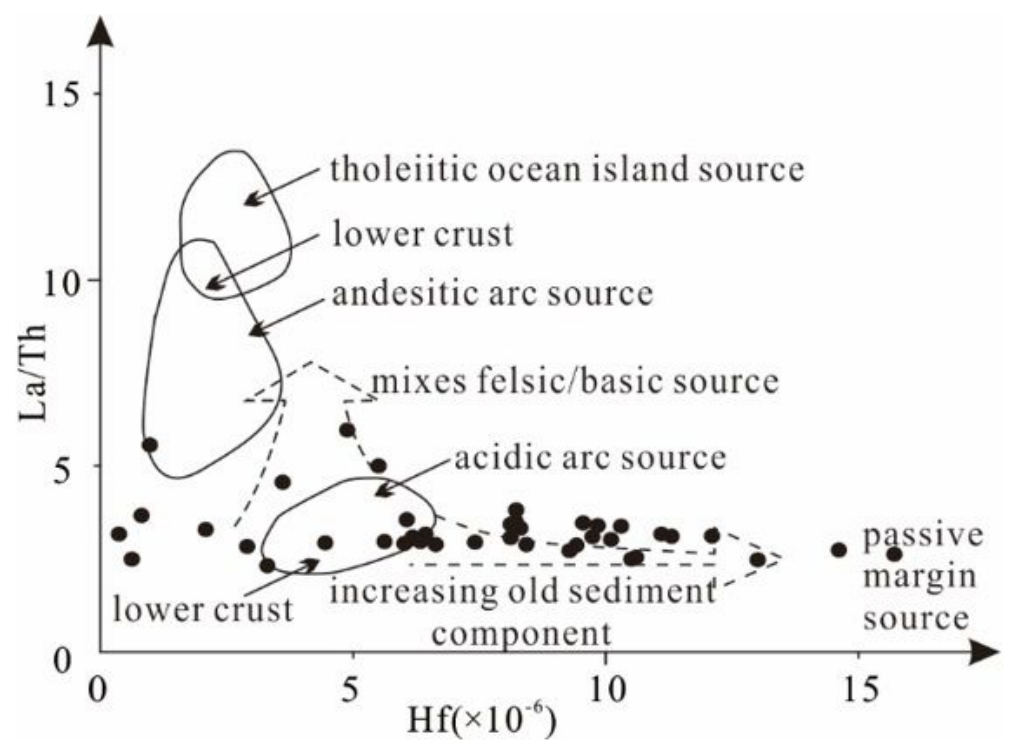

Figure 5

La/Th-Hf discriminatory plot (modified after Floyd et al.53) of late Cretaceous samples from the lower Zhoutian Formation, Jitai Basin. 